\title{
GEOMETRIC AND ANALYTIC INTERPRETATION OF ORTHOSCHEME AND LAMBERT CUBE IN EXTENDED HYPERBOLIC SPACE
}

\author{
Yunhi Cho AND Hyuk Kim
}

\begin{abstract}
We give a geometric proof of the analyticity of the volume of a tetrahedron in extended hyperbolic space, when vertices of the tetrahedron move continuously from inside to outside of a hyperbolic space keeping every face of the tetrahedron intersecting the hyperbolic space. Then we find a geometric and analytic interpretation of a truncated orthoscheme and Lambert cube in the hyperbolic space from the viewpoint of a tetrahedron in the extended hyperbolic space.
\end{abstract}

\section{Introduction}

The volume of a tetrahedron in the hyperbolic space is a bit complicated analytic function of dihedral angles (see [4], [11]). If we analytically continue its dihedral angles so that the vertices of tetrahedron move out crossing the ideal boundary, the volume function still gives us complex numbers as analytic continuations of volume. In fact, this function has a singularity exactly when a vertex passes through the ideal boundary and then becomes multi-valued after that point. In this paper, we want to investigate carefully the geometric meaning of real and imaginary part of this complex numbers appeared as branches.

The best way of describing these is to use the extended model studied in [6] (as a remark, [2] and [8] implicitly showed the strong evidence for the existence of 2 and 3-dimensional extended hyperbolic space, even though the paper [8] only considered hyperbolic space itself). The extended hyperbolic space is the projective space obtained from the Minkowski space with the induced metric where the sign of metric is changed for the Lorentzian part so that the sectional curvature is always equal to -1 . The metric is singular along the ideal boundary and for a unified treatment of these two spaces we need a way to define a

Received October 3, 2012; Revised March 1, 2013.

2010 Mathematics Subject Classification. 51M10, 51M25, 53B30.

Key words and phrases. hyperbolic space, volume, analytic continuation.

The second author was supported by the Korea Research Foundation(KRF) grant funded by the Korea government(MEST)(No. 20120001310). 
volume of a geometric object lying across the boundary. We used two different methods to achieve this goal. One way is to perturb the metric by replacing 1 by $d_{\epsilon}=1-\epsilon i$ in the denominator so that the metric becomes complex nonsingular metric, and then compute the volume and take a limit as $\epsilon \rightarrow 0$. The other way is an integration using polar coordinates and at the last integration in the radial direction we take a clockwise contour integral to avoid the singularity. And we showed these two methods give the same result. It is also shown that the $d_{\epsilon}=1+\epsilon i$ approximation corresponds to counterclockwise contour integration. The contour integrations also give us countably many different values depending on the contour types.

For a hyperbolic tetrahedron, if we continuously move vertices out from inside to outside of the hyperbolic space $\mathbb{H}^{3}$ keeping every edge of the tetrahedron intersecting $\mathbb{H}^{3}$ (the more general condition of keeping every face intersecting $\mathbb{H}^{3}$ is also considered in this paper), then the volume of this ultra tetrahedron in the extended space is expressed by the same analytic multi-valued function. Only when the moving vertex is on the ideal boundary $\partial \mathbb{H}^{3}$, the volume function is not analytic but continuous at the point.

Also, we obtain the following nice geometric interpretation (a generalization of the results of Kellerhals [8] and Usijima [13]) for the multi-valued analytic tetrahedron volume formula $f$ :

$$
\begin{aligned}
f= & \operatorname{Re} f+i \operatorname{Im} f \\
= & \text { volume of truncated tetrahedron } \\
& +\frac{\pi i}{4} \text { (total area of the truncated faces), }
\end{aligned}
$$

for clockwise analytic continuation of $f$ and the clockwise definition of the extended hyperbolic space, or

$f=$ volume of truncated tetrahedron $-\frac{\pi i}{4}$ (total area of the truncated faces),

for counterclockwise analytic continuation of $f$ and the counterclockwise definition of the extended hyperbolic space. The truncated tetrahedron in (1) is obtained from a tetrahedron by polar truncation at the vertices lying outside the hyperbolic space. For other branches, we get a generalized version of (1) (see (10)).

In fact, many mathematicians confronted this kind of choice problem from a multi-valued volume, it seems very difficult to find a suitable choice from a multi-valued analytic volume function. However the volume of the general tetrahedron with vertices beyond the ideal boundary in the extended hyperbolic space takes only one value among the multi-values with a suitable contour choice, or the choice of $d_{\epsilon}$. This shows one useful aspect of extended hyperbolic space, i.e., extended hyperbolic space always gives a unique consistent volume choice among multi-values. 
In addition, we can easily imagine that the analytic volume formula of a hyperbolic tetrahedron could be applicable to a general tetrahedron wherever it is positioned in extended hyperbolic space. The analyticity of the volume formula for a general tetrahedron across the hyperbolic ideal boundary in extended space will be treated in a future work.

From the natural geometric viewpoint, we are convinced that our result and [7] will show an interesting relation between extended space theory and scissors congruence theory.

Lastly, we give a simple application of our analyticity result to the volumes of orthoschemes, Lambert cubes, and prisms. In particular, we derive the following formula (see Fig. 11 and Example 4.7) for the analytic orthoscheme volume formula $f$ :

$$
\begin{aligned}
f= & \operatorname{Re} f+i \operatorname{Im} f \\
= & (\operatorname{vol}\langle 235678910\rangle+\operatorname{vol}\langle 1478\rangle)+(\operatorname{vol}\langle 15678\rangle+\operatorname{vol}\langle 478910\rangle) \\
= & (\text { volume of a Lambert cube }+ \text { volume of a tetrahedron with } 2 \text { opposite } \\
& \text { non-rectangular dihedral angles })+(\text { volume of two half lunes with } \\
& \text { quadrangle sections }\langle 5678\rangle \text { and }\langle 78910\rangle),
\end{aligned}
$$

for either a clockwise or counterclockwise approach.

\section{Lobachevsky function and Vinberg formula}

The volumes of some polyhedra in the hyperbolic space $\mathbb{H}^{n}$ are functions of dihedral angles expressed in terms of Lobachevsky function.

Following Milnor [10], we define the Lobachevsky function by the formula

$$
\wedge(x)=-\int_{0}^{x} \log |2 \sin t| d t .
$$

The function $\wedge$ is odd, is periodic with period $\pi$, vanishes at points $\frac{n \pi}{2}$, reaches its maximum at the points $n \pi+\frac{\pi}{6}$, and reaches its minimum at the points $n \pi-\frac{\pi}{6}$. It is real analytic everywhere except at the points $n \pi$, where its derivative tends to $\infty$.

The function $\wedge(x)$ can be extended from the interval $(0, \pi)$ to a multi-valued analytic function $\Lambda$ in the complex plane, branching at the points $n \pi$. The function $\Lambda$ is connected to the Euler dilogarithm function,

$$
L i_{2}(z)=\sum_{n=1}^{\infty} \frac{z^{n}}{n^{2}}, \quad|z| \leq 1,
$$

by the relation (see $\S 10.4$ of [12])

$$
L i_{2}\left(e^{2 i z}\right)=\frac{\pi^{2}}{6}-z(\pi-z)+2 i \Lambda(z), \quad \operatorname{Im} z>0 .
$$

By applying the Schwartz reflection principle to $\Lambda\left(z+\frac{\pi}{2}\right)$ and $i \Lambda\left(i z+\frac{\pi}{2}\right)$, we get $\Lambda\left(z+\frac{\pi}{2}\right)+\Lambda\left(-z+\frac{\pi}{2}\right)=0$, where the function $\Lambda$ is calculated on $\operatorname{Im} z<0$. Here 
the analytic multi-valued function $\Lambda(z)$ is uniquely defined on the branch cut $\mathbb{C}-((-\infty, 0) \cup(\pi, \infty))$. Furthermore, we easily obtain $\Lambda(z+\pi)=\Lambda(z)+z \pi i$, $\operatorname{Im} z>0$, from the formula (3). Hence $0=\Lambda(-z)+\Lambda(z+\pi)=\Lambda(-z)+\Lambda(z)+$ $z \pi i, \operatorname{Im} z>0$, so we get $\Lambda(-z)+\Lambda(z)=-z \pi i, \operatorname{Im} z>0$.

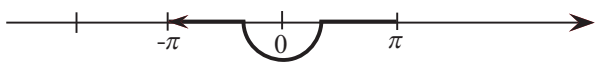

\section{FIGURE 1}

If we send $z$ to $(0, \pi)$ from the upper half plane, then $-z$ goes to $(-\pi, 0)$ from the lower half plane. Hence we want to determine the branch value of $\Lambda(x), x \in(-\pi, 0)$, as the analytic continuation along the contour depicted in Fig. 1. Therefore we conclude $\Lambda(-x)=-\Lambda(x)-\pi x i, x \in(0, \pi)$, and $\Lambda(x)=-\Lambda(-x)+\pi x i, x \in(-\pi, 0)$, i.e.,

$$
\begin{aligned}
\Lambda(x) & =-\wedge(-x)+\pi x i, \quad x \in(-\pi, 0), \\
& =\wedge(x)+\pi x i .
\end{aligned}
$$

This relation shows that the difference between $\wedge(x)$ and $\Lambda(z)$ on the clockwise contour choice (see Fig. 1) for $\Lambda(x), x \in(-\pi, 0)$, gives a real part $\wedge(x)$ and an imaginary part $\pi x i$ among multi-values. In this paper, we will use this branch value determined by the clockwise contour choice. If the contour direction is changed to a counterclockwise contour (going through upper half plane), then we can easily show that $\Lambda(x), x \in(-\pi, 0)$, has a real part $\wedge(x)$ and an imaginary part $-\pi x i$.

Note also that the dilogarithm function $L i_{2}(z)$ pinches up $2 \pi i \log z$ additionally as a branch effect for each clockwise contour around $z=1$ starting and ending at $z$. So $\Lambda(z)$ has a $\frac{2 \pi i}{2 i} \log e^{2 i z}=2 \pi i z$ branch effect for each clockwise contour around $z=0$. Whenever we consider $\Lambda(z)$ with one more clockwise full rotation contour around $z=0$, we have to add up $2 \pi i z$ for fixed $z$.

For a hyperbolic tetrahedron with all its vertices at infinity, the relation between dihedral angles implies that the dihedral angles corresponding to opposite edges are equal. Denoting the different dihedral angles of such a tetrahedron by $\alpha, \beta, \gamma$, one also has $\alpha+\beta+\gamma=\pi$. The volume of such a tetrahedron $T$ is easily expressed as

$$
\operatorname{vol}(T)=\wedge(\alpha)+\wedge(\beta)+\wedge(\gamma)
$$

All of the dihedral angles for a hyperbolic tetrahedron $T$ with three vertices at infinity are expressed in terms of the dihedral angles $\alpha, \beta, \gamma$ at the finite vertex (see Fig. 2). The volume of such a tetrahedron is (see [1] or [14])

(6) $2 \operatorname{vol}(T)=\wedge(\alpha)+\wedge(\beta)+\wedge(\gamma)+\wedge\left(\alpha_{1}\right)+\wedge\left(\beta_{1}\right)+\wedge\left(\gamma_{1}\right)-\wedge\left(\frac{\alpha+\beta+\gamma-\pi}{2}\right)$,

where $\alpha_{1}=\frac{\pi+\alpha-\beta-\gamma}{2}, \beta_{1}=\frac{\pi-\alpha+\beta-\gamma}{2}, \gamma_{1}=\frac{\pi-\alpha-\beta+\gamma}{2}$. 


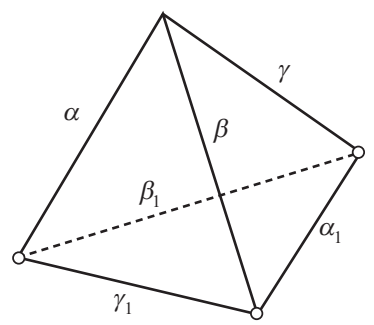

Figure 2

\section{General tetrahedron volume formula with three ideal vertices}

To consider the geometry of a general tetrahedron in projective space, we will use the Kleinian metric on the usual affine coordinates chart containing hyperbolic space as the unit ball. Then we can make an extended hyperbolic space from the Kleinian metric on the affine charts (see [6]); see the many elementary properties of the space in [6] and [3].

There are two kinds of extended hyperbolic space: the extended Kleinian model $K^{n}$ (or projective space model $\mathbb{R} P_{H}^{n}$ ) and the hyperbolic sphere model $\mathbb{S}_{H}^{n}$; these extended hyperbolic spaces have generalized volume measures ( $\mu$ measure in [6] and $\mathrm{vol}_{H}$ in [3]) with complex values. In fact, the $\mu$-measure and vol ${ }_{H}$ are the same measure.

From the definition of the volume in the extended hyperbolic space $\mathbb{S}_{H}^{n}$, we can show one of the similarities between $\mathbb{S}_{H}^{n}$ and $\mathbb{S}^{n}$, that is, vol ${ }_{H}\left(\mathbb{S}_{H}^{n}\right)=$ $i^{n} \cdot \operatorname{vol}\left(\mathbb{S}^{n}\right)$ (see [6]). If we change the contour type of the integral, we have a different relation between $\operatorname{vol}_{H}\left(\mathbb{S}_{H}^{n}\right)$ and $\operatorname{vol}\left(\mathbb{S}^{n}\right)$.

When $n=3$, we have $\operatorname{vol}_{H}\left(\mathbb{S}_{H}^{3}\right) \equiv i^{3} \cdot \operatorname{vol}\left(\mathbb{S}^{3}\right)\left(\bmod 2 i^{3} \cdot \operatorname{vol}\left(\mathbb{S}^{3}\right)\right)$. More precisely, whenever we consider $\mathbb{S}_{H}^{3}$ with one more full rotation clockwise contour, we have to add up $2 i^{3} \cdot \operatorname{vol}\left(\mathbb{S}^{3}\right)=-4 \pi^{2} i$.

Naturally, the isometry group $P O(n, 1)$ can be expected to preserve the complex volume. In fact, we have the following proposition (see Proposition 3.2 in [6] for a proof, and see Theorem 3.6 in [5] for a more delicate boundary condition).

Proposition 3.1. Let $U$ be a domain with a piecewise analytic boundary transversal to $\partial \mathbb{H}^{n}$ in extended hyperbolic space. Then $\operatorname{vol}_{H}(U)$ has a welldefined finite value and $\operatorname{vol}_{H}(g(U))=\operatorname{vol}_{H}(U)$ for each $g \in P O(n, 1)$.

In this paper, we need only five things among the geometric properties of the extended hyperbolic space developed in [6] or [3]: an important and natural property Proposition 3.1, which will be used implicitly in this paper; the volume of a trihedron Tri $(A, B, C)$ with dihedral angles $A, B, C$ is represented as (see [6])

$$
\operatorname{vol}_{H}(\operatorname{Tri}(A, B, C))=\frac{\pi}{2} i(\pi-A-B-C),
$$


the basic angle definition (see Definitions 3.3, 3.6 and Fig. 12 in [3]); generalized trigonometry (see Theorem 4.13 in [3]); and the symmetric property (see Theorem 4.1 in [6]). Henceforth, we will use $\operatorname{vol}(U)$ instead of $\operatorname{vol}_{H}(U)$ for the

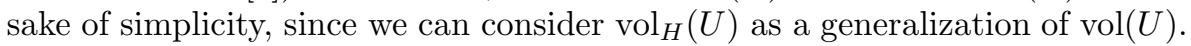

Even though we draw a tetrahedron across the ideal boundary, we mostly deal with the magnitudes of angles and lengths lying inside the hyperbolic space in the paper, so we need not use the generalized definition (see [6] or [3]) of angles and lengths for most cases in the paper. As an exception, we deal with pure imaginary angles in the Lambert cube case and in Example 4.9.

We know the volume formula (6) for a hyperbolic tetrahedron with three vertices at infinity and one hyperbolic vertex by Vinberg's geometric technique. Now, we derive and show the volume formula for a hyperbolic tetrahedron with three vertices at infinity and one Lorentzian vertex below.

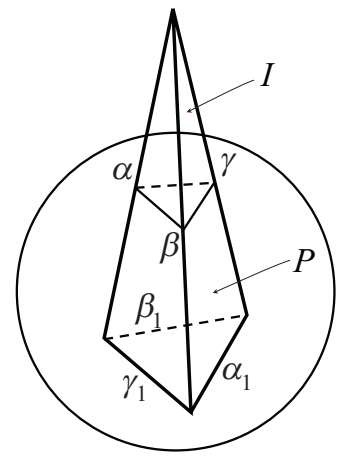

Figure 3

From Ushijima's result [13], the volume of the polyhedron $P$ obtained by the truncation of the dual plane of the Lorentzian vertex of the tetrahedron with three ideal vertices and one Lorentzian vertex (see $P$ in Fig. 3) is represented by the real part of an analytic function $f$ that coincides with the volume of a tetrahedron in the usual hyperbolic tetrahedron case.

Hence, we need only to change $\wedge$ to $\Lambda$ in the formula (6), where we need to check one more fact that all the values inside the parenthesis must be inside $(0, \pi)$ in the usual hyperbolic tetrahedron case. Also, note that the two functions $\Lambda$ and $\wedge$ have the same real values without imaginary parts for $\alpha, \beta, \gamma, \alpha_{1}, \beta_{1}, \gamma_{1}$, but $\Lambda\left(\frac{\alpha+\beta+\gamma-\pi}{2}\right) \neq \wedge\left(\frac{\alpha+\beta+\gamma-\pi}{2}\right)$. If we pull the non-ideal vertex out to outside $\mathbb{H}^{n}$, then the value $\frac{\alpha+\beta+\gamma-\pi}{2}$ will change from + to - , so we get

$$
\Lambda\left(\frac{\alpha+\beta+\gamma-\pi}{2}\right)=\wedge\left(\frac{\alpha+\beta+\gamma-\pi}{2}\right)+\pi i\left(\frac{\alpha+\beta+\gamma-\pi}{2}\right)
$$


for the clockwise contour choice (see Fig. 1) by formula (4). Formula (8) also shows the continuity property of $\Lambda\left(\frac{\alpha+\beta+\gamma-\pi}{2}\right)$ at the singular points.

Therefore, we know

$$
\begin{aligned}
& 2 \operatorname{vol}(P) \\
= & \operatorname{Re}\left(\Lambda(\alpha)+\Lambda(\beta)+\Lambda(\gamma)+\Lambda\left(\alpha_{1}\right)+\Lambda\left(\beta_{1}\right)+\Lambda\left(\gamma_{1}\right)-\Lambda\left(\frac{\alpha+\beta+\gamma-\pi}{2}\right)\right) \\
= & \wedge(\alpha)+\wedge(\beta)+\wedge(\gamma)+\wedge\left(\alpha_{1}\right)+\wedge\left(\beta_{1}\right)+\wedge\left(\gamma_{1}\right)-\wedge\left(\frac{\alpha+\beta+\gamma-\pi}{2}\right),
\end{aligned}
$$

where $\alpha_{1}=\frac{\pi+\alpha-\beta-\gamma}{2}, \beta_{1}=\frac{\pi-\alpha+\beta-\gamma}{2}, \gamma_{1}=\frac{\pi-\alpha-\beta+\gamma}{2}$.

Furthermore, we know the trihedron volume formula (7), so we obtain the volume of the polyhedron $I$, which is half the volume of a trihedron. The trihedron has three dihedral angles $\alpha, \beta, \gamma$, so we get the following formula

$$
\operatorname{vol}(I)=\frac{\pi}{4} i(\pi-\alpha-\beta-\gamma)
$$

Finally, we conclude the following formula by (8):

$$
\begin{aligned}
& 2(\operatorname{vol}(P)+\operatorname{vol}(I)) \\
= & \wedge(\alpha)+\wedge(\beta)+\wedge(\gamma)+\wedge\left(\alpha_{1}\right)+\wedge\left(\beta_{1}\right)+\wedge\left(\gamma_{1}\right)-\wedge\left(\frac{\alpha+\beta+\gamma-\pi}{2}\right) \\
& +\frac{\pi}{2} i(\pi-\alpha-\beta-\gamma) \\
= & \Lambda(\alpha)+\Lambda(\beta)+\Lambda(\gamma)+\Lambda\left(\alpha_{1}\right)+\Lambda\left(\beta_{1}\right)+\Lambda\left(\gamma_{1}\right)-\Lambda\left(\frac{\alpha+\beta+\gamma-\pi}{2}\right) .
\end{aligned}
$$

Additionally, we remark that we gave the volume of $I$ as a unique value. Basically, there is a difficulty in the valuation of $I$, since the volume of $I$ can take multi-values from the viewpoint of the analytic function approach (using the Schläfli formula), so we have to choose one proper value among multivalues. However, we fixed the analytic continuation direction as the clockwise direction for the function $\Lambda$ and the extended hyperbolic space, so we can get only one value for each geometric object in hyperbolic space. The unique value property is one good aspect of extended hyperbolic space.

We conclude the following theorem.

Theorem 3.2. For a given tetrahedron inside $\mathbb{H}^{3}$ with three ideal vertices, if we move the non-ideal vertex out continuously from inside to outside of $\mathbb{H}^{3}$, then the volume formula is expressed by an analytic multi-valued function (9). The analytic function will take a unique value for a contour choice, and only when the moving vertex is on the ideal boundary $\partial \mathbb{H}^{3}$, the function is not analytic but continuous at that point.

Remark 3.3. For a counterclockwise analytic continuation direction for the function $\Lambda$ and the extended hyperbolic space, we can also obtain the same formula (9). However, we have the following different formulas instead of (8) 
and the volume of $I$,

$$
\begin{aligned}
\Lambda\left(\frac{\alpha+\beta+\gamma-\pi}{2}\right) & =\wedge\left(\frac{\alpha+\beta+\gamma-\pi}{2}\right)-\pi i\left(\frac{\alpha+\beta+\gamma-\pi}{2}\right), \\
\operatorname{vol}(I(A, B, C)) & =-\frac{\pi}{4} i(\pi-A-B-C)
\end{aligned}
$$

The difference of \pm sign does not have any effect on formula (9).

In order to handle a more general contour case, we have to find the contour effect of $\Lambda\left(\frac{\alpha+\beta+\gamma-\pi}{2}\right)$ and $\operatorname{vol}(I(A, B, C))$. The polyhedron $I$ has a vertex in the Lorentzian part, and the bottom triangle $S$ is located in the hyperbolic part (see Fig. 3), so we can calculate the volume of $I$ by using contour integration and the multiple integration of Theorem 4.1 of [6].

By contour integration, we obtain

$$
\begin{aligned}
\operatorname{vol}(I) & =\operatorname{vol}\left(\mathbb{S}_{H}^{3}\right) \times \frac{\operatorname{vol}(S)}{\operatorname{vol}\left(\mathbb{S}_{H}^{2}\right)}=\frac{k \pi i S}{4}=\frac{k \pi i}{4}(\pi-A-B-C), \\
k & =\ldots,-5,-3,-1,1,3,5, \ldots
\end{aligned}
$$

Whenever we consider one more full clockwise rotation contours for vol $(I)$, we have to add up $\frac{\pi i}{2}(\pi-A-B-C)$ for the given value. We already explained the contour effect of $\Lambda(z)$ in Section 2. The full rotation effect of $\Lambda\left(\frac{\alpha+\beta+\gamma-\pi}{2}\right)$ is $2 \pi i\left(\frac{\alpha+\beta+\gamma-\pi}{2}\right)=\pi i(\alpha+\beta+\gamma-\pi)$.

Finally, we have the following relation for any contour ( $k$ an odd integer):

$$
\begin{aligned}
& \operatorname{vol}(P)+\operatorname{vol}(I) \\
= & \frac{1}{2}\left(\wedge(\alpha)+\cdots+\wedge\left(\gamma_{1}\right)-\wedge\left(\frac{\alpha+\beta+\gamma-\pi}{2}\right)+\frac{k \pi i}{2}(\pi-\alpha-\beta-\gamma)\right), \\
= & \frac{1}{2}\left(\Lambda(\alpha)+\Lambda(\beta)+\Lambda(\gamma)+\Lambda\left(\alpha_{1}\right)+\Lambda\left(\beta_{1}\right)+\Lambda\left(\gamma_{1}\right)-\Lambda\left(\frac{\alpha+\beta+\gamma-\pi}{2}\right)\right) .
\end{aligned}
$$

Therefore, we conclude that if we fix the contour type in the calculation of the extended hyperbolic space and $\Lambda(z)$, then the volume of a tetrahedron of the type of Theorem 3.2 and the analytic function (9) have exactly the same value among the multi-values.

In the Appendix, we provide another elementary proof of Theorem 3.2. In this proof, we do not use Ushijima's result, but only elementary geometric properties of extended hyperbolic space.

\section{Volume formula of a general tetrahedron whose vertices are lying outside $\mathbb{H}^{3}$}

We extend its 5 edges of a given compact hyperbolic tetrahedron to ideal points, as given in Fig. 4, to obtain an ideal polyhedron with six vertices. This ideal polyhedron was already handled in [4].

The volume of the given tetrahedron $\langle a b c d\rangle$ can be derived from the following formula:

$$
\operatorname{vol}\langle a b c d\rangle=\operatorname{vol}\langle a 123\rangle+\operatorname{vol}\langle b 456\rangle+\operatorname{vol}\langle c d 2356\rangle-\operatorname{vol}\langle 123456\rangle .
$$



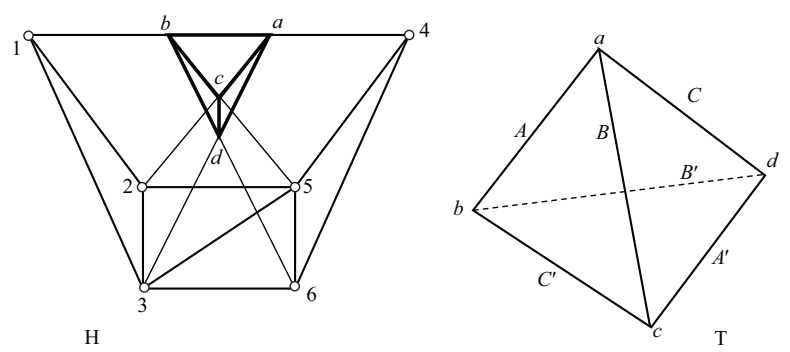

Figure 4
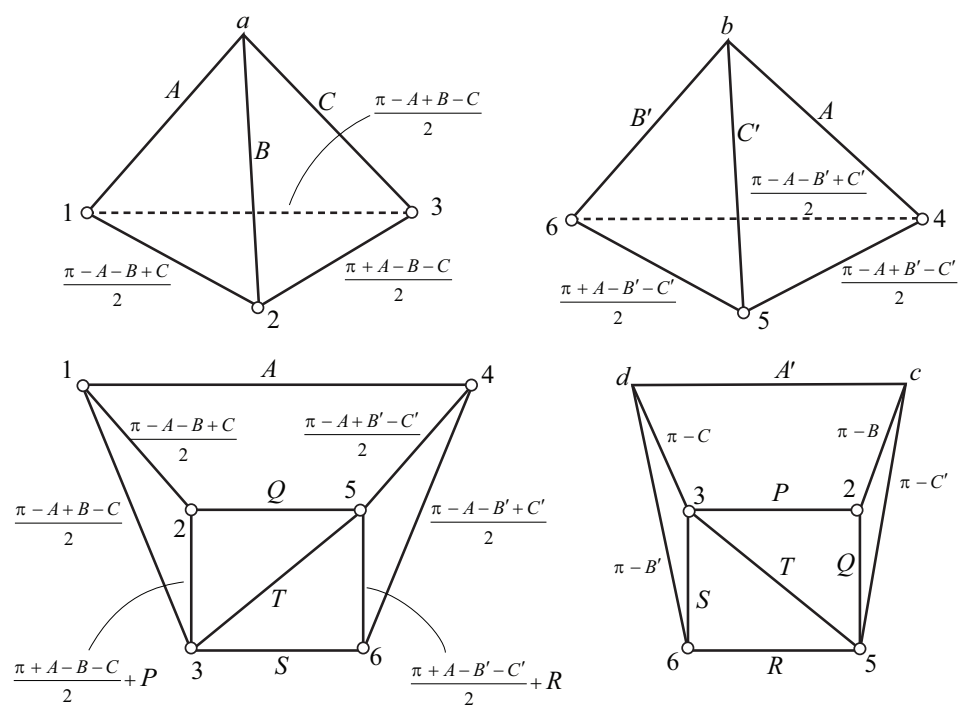

Figure 5

The dihedral angles of each piece are shown in Fig. 5, where

(12) $P+Q=B, R+S=B^{\prime}, Q+R+T=\pi+C^{\prime}, P+S+T=\pi+C$,

and we obtain one more relation from the Gram matrix condition of the polyhedron $\langle c d 2356\rangle$ with eliminations of one row and one column (see [4]),

$$
\left|\begin{array}{ccccc}
1 & -\cos A^{\prime} & -\cos P & \cos B & \cos C \\
-\cos A^{\prime} & 1 & \cos (R+T) & \cos C^{\prime} & \cos B^{\prime} \\
-\cos P & \cos (R+T) & 1 & -\cos Q & \cos (S+T) \\
\cos B & \cos C^{\prime} & -\cos Q & 1 & -\cos A \\
\cos C & \cos B^{\prime} & \cos (S+T) & -\cos A & 1
\end{array}\right|=0
$$


It is well known (see [11]) that the system (12), (13) has two solutions $\left(P_{1}, Q_{1}, R_{1}, S_{1}, T_{1}\right)$ and $\left(P_{2}, Q_{2}, R_{2}, S_{2}, T_{2}\right)$ induced from a quadratic equation, and we also know (see [4]) that

$$
\begin{aligned}
2 \operatorname{vol}(T)= & \wedge\left(P_{1}\right)-\wedge\left(Q_{1}\right)+\wedge\left(R_{1}\right)-\wedge\left(S_{1}\right) \\
& -\wedge\left(\frac{B-C-A+\pi}{2}-Q_{1}\right)+\wedge\left(\frac{A^{\prime}-B-C^{\prime}+\pi}{2}+Q_{1}\right) \\
& +\wedge\left(\frac{B^{\prime}-C-A^{\prime}+\pi}{2}-R_{1}\right)-\wedge\left(\frac{A-B^{\prime}-C^{\prime}+\pi}{2}+R_{1}\right) \\
& -\wedge\left(P_{2}\right)+\wedge\left(Q_{2}\right)-\wedge\left(R_{2}\right)+\wedge\left(S_{2}\right) \\
& +\wedge\left(\frac{B-C-A+\pi}{2}-Q_{2}\right)-\wedge\left(\frac{A^{\prime}-B-C^{\prime}+\pi}{2}+Q_{2}\right) \\
& -\wedge\left(\frac{B^{\prime}-C-A^{\prime}+\pi}{2}-R_{2}\right)+\wedge\left(\frac{A-B^{\prime}-C^{\prime}+\pi}{2}+R_{2}\right),
\end{aligned}
$$

where the solutions $\left(P_{1}, Q_{1}, R_{1}, S_{1}, T_{1}\right)$ and $\left(P_{2}, Q_{2}, R_{2}, S_{2}, T_{2}\right)$ of the equations (12) and (13) are chosen so that the value of (14) is positive.

We can represent (14) simply as

$$
\begin{aligned}
\operatorname{vol}(T)= & f\left(A, B, C, A^{\prime}, B^{\prime}, C^{\prime}, P_{1}, Q_{1}, R_{1}, S_{1}, T_{1}\right) \\
& -f\left(A, B, C, A^{\prime}, B^{\prime}, C^{\prime}, P_{2}, Q_{2}, R_{2}, S_{2}, T_{2}\right) .
\end{aligned}
$$

Note that the function $f$ is a continuous and real analytic function with singularity, and $\operatorname{vol}(T) \neq 0$ for all non-degenerate hyperbolic tetrahedra, which implies that the quadratic equation always has two distinct solutions. Hence, we can conclude that the solutions $\left(P_{1}, Q_{1}, R_{1}, S_{1}, T_{1}\right)$ and $\left(P_{2}, Q_{2}, R_{2}, S_{2}, T_{2}\right)$ are continuous functions of variables $A, B, C, A^{\prime}, B^{\prime}, C^{\prime}$, and that each solution has a unique expression.

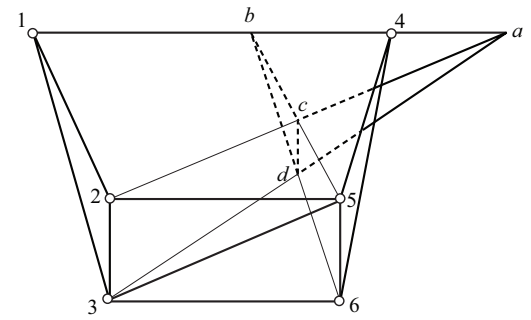

FiguRe 6

Now we consider a more general situation. Let us imagine that the hyperbolic point $a$ in Fig. 4 is continuously passing through the ideal boundary to be in the Lorentzian part, as in Fig. 6. Then, we get a tetrahedron $\langle a b c d\rangle$ with 3 hyperbolic points and 1 Lorentzian point. Hence the volume of the one vertex outside the tetrahedron $\langle a b c d\rangle$ is also represented by the same formula 
(11) and the dihedral angles of each piece also have the same expression as in Fig. 5 with the same conditions (12), (13).

Note that the solution $(P, Q, R, S, T)$ we want comes from the genuine shape of the polyhedron $\langle c d 2356\rangle$, so all terms inside of the Lobachevsky functions $\wedge$ appearing in the expressions of $\operatorname{vol}\langle b 456\rangle, \operatorname{vol}\langle c d 2356\rangle, \operatorname{vol}\langle 123456\rangle$ have values in $(0, \pi)$. However, the inside value $\frac{\alpha+\beta+\gamma-\pi}{2}$ of $\wedge\left(\frac{\alpha+\beta+\gamma-\pi}{2}\right)$ appearing in $\operatorname{vol}\langle a 123\rangle$ lies in $(-\pi, \pi)$, so it must pass through the singular point 0 , but if all real Lobachevsky functions are changed to complex analytic Lobachevsky functions, then every two corresponding polyhedra in Figs. 4 and 6 have the same analytic representation by Theorem 3.2. In fact, we need one more argument, that is, vol $\langle c d 2356\rangle$ to be analytically represented. The polyhedron $\langle c d 2356\rangle$ with given dihedral angles variables could be divided into three tetrahedra with six unknown variables. Then ideal conditions (which give 4 formulas) and the coincidence conditions (which give 2 formulas) between two facial angles of pieces at the finite vertex suffice the remaining six conditions. We can show that the six unknown variables are uniquely analytically represented. Therefore $\operatorname{vol}\langle c d 2356\rangle$ is analytically represented.

Now we will explain property of volume continuity (see Fig. 7) on extended hyperbolic space.

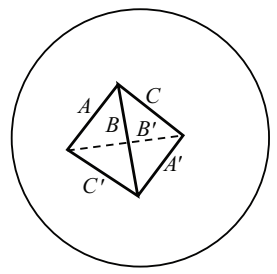

(a)

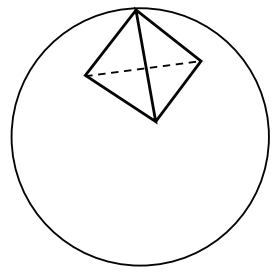

(b)

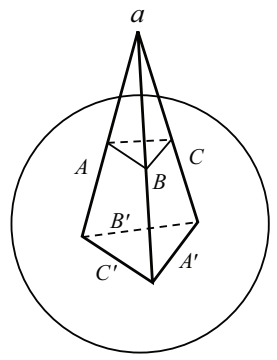

(c)

FiguRE 7

From the compact case Fig. 7(a) to the one ideal vertex case Fig. 7(b), the continuity of volume is well known. If we divide the tetrahedron shown in Fig. 7(c) into two pieces by cutting, using a dual plane $a^{\perp}$, then the upper part has a volume represented by $\frac{\pi i}{4}(\pi-A-B-C)$, so the value moves continuously from (b) to (c), and the lower part naturally has the continuous volume change property as well. One can imagine three vertices at the triangle $a^{\perp} \cap\langle a b c d\rangle$ in (c) go to a single ideal point continuously in (b).

There is one more thing to explain from the property of volume continuity. While one vertex is moving from inside to outside, we have to show that the choice of $(P, Q, R, S, T)$ is uniquely expressed by one formula of variables 
$A, B, C, A^{\prime}, B^{\prime}, C^{\prime}$ between two solutions of a quadratic equation. This is confirmed by the continuity of the volume of the tetrahedron as one vertex moves freely from inside to outside. The volume of the tetrahedron never goes to 0 , so two choices of the solution always give different values, and hence there are two leaves (non-intersecting each other) of values for their choices.

Consequently we can conclude the following theorem.

Theorem 4.1. For a hyperbolic tetrahedron with six dihedral angle variables, if we move one vertex out continuously from inside to outside of $\mathbb{H}^{3}$, then the volume formula is expressed by the same analytic multi-valued function. This analytic function takes a unique value determined by a clockwise (counterclockwise resp.) contour choice and this choice coincides with the volume of the tetrahedron in the fixed extended hyperbolic space choice coming from the clockwise (counterclockwise resp.) construction. When only the moving vertex is on the ideal boundary $\partial \mathbb{H}^{3}$, the volume function is not analytic but is continuous at the point.

Remark 4.2. For this theorem, we can simply say

a multi-valued analytic function $f:=\operatorname{vol}($ tetrahedron)

which implies that

$f=$ volume of truncated tetrahedron + half volume of trihedron.

Note that $f$ takes different values for clockwise or counterclockwise contour, also the trihedron has different values:

$$
\operatorname{vol}(\operatorname{Tri}(A, B, C))= \pm \frac{\pi}{2} i(\pi-A-B-C),
$$

where for clockwise continuation we choose + , and for counterclockwise continuation we choose - .

Now we consider more general cases. If other vertices move from inside to outside of $\mathbb{H}^{3}$ with the condition that every edge always intersects $\mathbb{H}^{3}$ while the vertices are moving, then we can show a result similar to that of Theorem 4.1 by the following argument.

Now we always assume the edge intersection (to $\mathbb{H}^{3}$ ) condition above for all cases. If two vertices of the tetrahedron lie outside $\mathbb{H}^{3}$, then we can apply a similar method, as before. We remark that the only a difference between the two situations is that the vertex $b$ in Fig. 6 is located outside of the extension of edge $\langle 14\rangle$. However, this method cannot be applied to the cases of three or four Lorentzian vertices, so we will introduce a more general argument. Let us consider two tetrahedra, one ( $W$ in Fig. 8) has 4 hyperbolic points and the other ( $V$ in Fig. 8 ) has 3 or 4 Lorentzian vertices. Then we divide each tetrahedron into two pieces of tetrahedron with one equal dihedral angle $\frac{A}{2}$ (see Fig. 8). Here we should determine two dihedral angles $x$ and $y$ for two different cases. The vertex 3 becomes a hyperbolic point or a Lorentzian point in each case. 

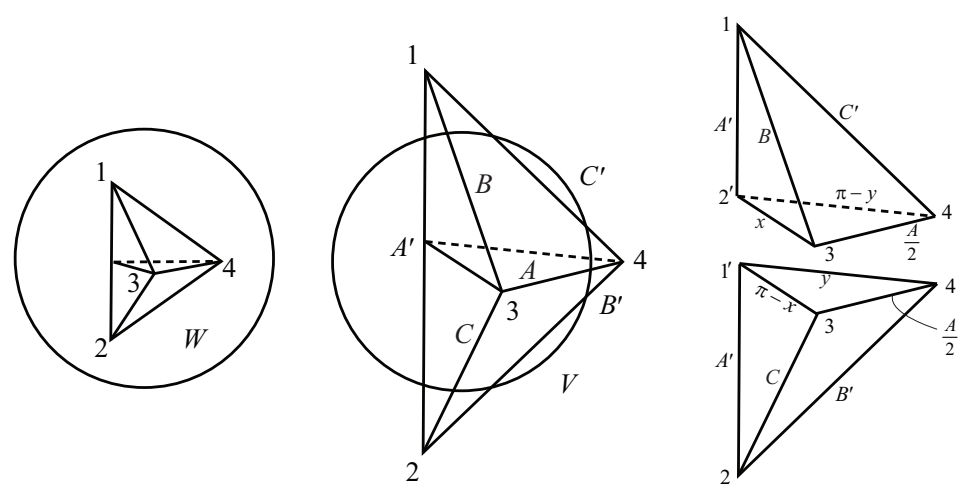

FIGURE 8

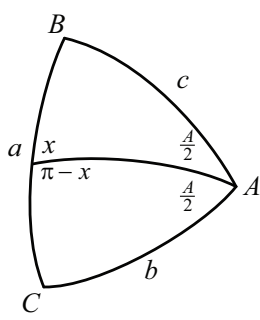

Spherical

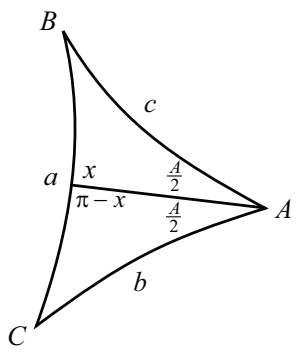

Hyperbolic

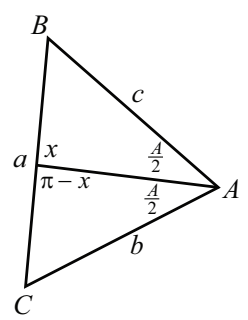

Euclidean

FiguRE 9

If vertex 3 is a hyperbolic point, then we obtain a spherical triangle with angles $A, B, C$ (see Fig. 9) by a small sphere cutting at vertex 3 . Then the dihedral angle $x$ becomes a subdivided angle $x$ in the spherical triangle (see Fig. 9), so the spherical cosine law induces two formulas

$$
\cos c=\frac{\cos A \cos B+\cos C}{\sin A \sin B} \quad \text { and } \quad \cos c=\frac{\cos \frac{A}{2} \cos B+\cos x}{\sin \frac{A}{2} \sin B} .
$$

Hence, we obtain

$$
\cos x=\frac{\sin \frac{A}{2}}{\sin A}(\cos A \cos B+\cos C)-\cos \frac{A}{2} \cos B .
$$

If vertex 3 is a Lorentzian point, then we obtain a hyperbolic triangle with angles $A, B, C$ (see Fig. 9) by slicing with the dual plane $3^{\perp}$. Then the angle 
$x$ is obtained using the hyperbolic cosine law, so we have

$$
\cosh c=\frac{\cos A \cos B+\cos C}{\sin A \sin B} \quad \text { and } \quad \cosh c=\frac{\cos \frac{A}{2} \cos B+\cos x}{\sin \frac{A}{2} \sin B} .
$$

Hence we obtain the same formula (15).

If vertex 3 is an ideal point, then we know $x=\pi-B-\frac{A}{2}$ which is induced from the formula (15) as well, with an additional condition $C=\pi-A-B$. We note that $\cos ^{-1} z=-i \log \left(z+\left(z^{2}-1\right)^{1 / 2}\right)$, and hence the angles $x=$ $\cos ^{-1}(\cdots)$ and $y=\cos ^{-1}(\cdots)$ are represented by analytic functions $x(A, B, C)$ and $y(A, B, C)$ of variables $A, B, C$ for all cases.

Therefore the tetrahedron $\langle 1234\rangle$ with dihedral angles $A, B, C, A^{\prime}, B^{\prime}, C^{\prime}$ in Fig. 8 is divided into two tetrahedra, and the corresponding angles of each piece have the same analytic representation for their original dihedral angles $A, B, C, A^{\prime}, B^{\prime}, C^{\prime}$. Precisely,

$$
\begin{aligned}
\operatorname{vol}\left(A, B, C, A^{\prime}, B^{\prime}, C^{\prime}\right)= & \operatorname{vol}\left(\frac{A}{2}, B, x(A, B, C), A^{\prime}, \pi-y\left(A, B^{\prime}, C^{\prime}\right), C^{\prime}\right) \\
& +\operatorname{vol}\left(\frac{A}{2}, B^{\prime}, y\left(A, B^{\prime}, C^{\prime}\right), A^{\prime}, \pi-x(A, B, C), C\right) .
\end{aligned}
$$

Then the two tetrahedra $\left\langle 12^{\prime} 34\right\rangle$ and $\left\langle 1^{\prime} 234\right\rangle$ from $V$ in Fig. 8 have 1 or 2 hyperbolic point and 3 or 2 Lorentzian points. We repeat this process for each piece of the tetrahedra to obtain 8 tetrahedral pieces from $V$ and $W$. Then their corresponding dihedral angles have the same analytic representation. Each piece of $V$ has 3 hyperbolic points and 1 Lorentzian point, and each piece of $W$ has 4 hyperbolic points naturally. Finally we conclude that the two original tetrahedra have the same analytic volume formula by comparing of the two corresponding subdivided tetrahedra. Note that the corresponding two subdivided tetrahedra have the same analytic volume representation by Theorem 4.1.

Consequently we have the following theorem.

Theorem 4.3. For a hyperbolic tetrahedron with six dihedral angle variables, if we move vertices out continuously from inside to outside of $\mathbb{H}^{3}$ keeping every edge of the tetrahedron intersecting the hyperbolic space $\mathbb{H}^{3}$, then the volume formula is expressed by the same analytic multi-valued function. This analytic function takes a unique value for a clockwise (counterclockwise resp.) contour choice, and this choice coincides with the volume of the tetrahedron of the fixed extended hyperbolic space defined from the clockwise (counterclockwise resp.) contour. When the moving vertex is on the ideal boundary $\partial \mathbb{H}^{3}$, the volume function is not analytic but is continuous at the point.

Remark. Under the same geometric conditions of Theorem 4.3, if we are able to analyze formula (14) (additionally we need an analytic change of $\wedge \rightarrow \Lambda$ in the formula), then Theorem 4.3 can be proved more directly without using Theorem 3.2 and Theorem 4.1. 
For example, if we move the vertex $a$ in Figure 4 to outside of $\mathbb{H}^{3}$, then we can easily guess that $P_{2}$ and $\frac{B-C-A+\pi}{2}-Q_{2}$ in formula (14) change their signs, and we get the following imaginary value from the clockwise contour effect of $\Lambda$ :

$$
\pi i\left(-P_{2}+\frac{B-C-A+\pi}{2}-Q_{2}\right)=\pi i\left(\frac{\pi-A-B-C}{2}\right) .
$$

Hence, this shows Theorem 4.1 more directly. However, in order to induce the same results as the Theorem, we have to prove $-\pi<P_{2}<0$ and $-\pi<$ $\frac{B-C-A+\pi}{2}-Q_{2}<0$ for the vertex $a$ outside case. Even though we can show that $P_{2}$ and $\frac{B-C-A+\pi}{2}-Q_{2}$ take real values from the technique of Lemma in $[11$, p. 384], it looks hard to prove the very complicated inequalities, in particular, $\frac{B-C-A+\pi}{2}-Q_{2}<0$. For other cases, we also need to show many complicated inequalities. Hence, we leave the direct proof as a problem to be solved.

From Theorem 4.3 we conclude that all analytic volume formulas $f$ of a hyperbolic tetrahedron (readers can see one nice formula in [11]) can also be applicable to a tetrahedron with vertices in the Lorentzian part with the edgeintersecting condition. Furthermore, we get the following corollary similar to Remark 4.2.

Corollary 4.4. We can see the following geometric interpretation of the analytic tetrahedron volume formula $f$ with the same condition as Theorem 4.3.

$$
\begin{aligned}
f & =\operatorname{Re} f+i \operatorname{Im} f \\
& =\text { volume of truncated tetrahedron }+ \text { half volume of trihedra } \\
& =\text { volume of truncated tetrahedron }+\frac{\pi i}{4} \text { (total area of the truncated faces). }
\end{aligned}
$$

Note that if we change the contour choice from clockwise to counterclockwise, then we have to change $\frac{\pi i}{4}$ to $-\frac{\pi i}{4}$ in (16). For a more general contour, we can also see the coincidence of the branch effect between the analytic function $f$ and the tetrahedral volume integration on $\mathbb{S}_{H}^{3}$. We obtain the following relation for an odd integer $k$ :

$f=$ volume of truncated tetrahedron $+\frac{k \pi i}{4}$ (total area of the truncated faces).

Although a formula similar to (16) was first mentioned in [6], where we used the volume of the tetrahedron instead of the complex analytic multi-valued function $f$, we could not derive the following corollary, because we could not analyze the multi-valued function $f$ and could not find the geometric meaning of that.

Corollary 4.5 (cf. Kellerhals [8], Usijima [13]). For the analytic tetrahedron volume formula $f$ with the same condition as Theorem 4.3, we have

$$
\operatorname{Re} f=\text { volume of truncated tetrahedra. }
$$


Note that Kellerhals also considered the Lambert cube case. The Lambert cube requires a more general situation than our result, namely, that one edge of a tetrahedron must be located entirely outside $\mathbb{H}^{3}$.

Now we can reconfirm Theorem 4.3 for the following examples. An orthoscheme $T(A, B, C)$ is a tetrahedron with three consecutive dihedral angles $A, B, C$ and three consecutive rectangular angles. We know that

$$
\begin{aligned}
\operatorname{vol}(T(A, B, C))= & \frac{1}{4}(\wedge(A+\delta)-\wedge(A-\delta)+\wedge(C+\delta)-\wedge(C-\delta) \\
& \left.-\wedge\left(\frac{\pi}{2}-B+\delta\right)+\wedge\left(\frac{\pi}{2}-B-\delta\right)+2 \wedge\left(\frac{\pi}{2}-\delta\right)\right),
\end{aligned}
$$

where $\delta=\tan ^{-1} \frac{\sqrt{\cos ^{2} B-\sin ^{2} A \sin ^{2} C}}{\cos A \cos C}$.

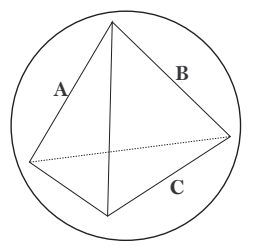

i)

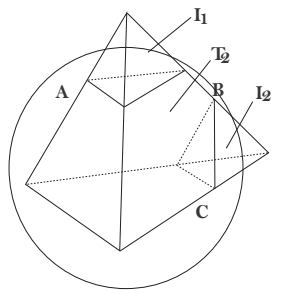

iii)

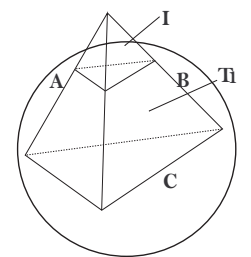

ii)

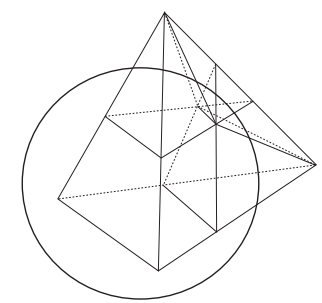

$i v)$

FIGURE 10

Kellerhals [8] showed that for one- or two-vertex truncated orthoschemes $T_{1}, T_{2}$, the volumes (see ii) and iii $\left.)\right) \operatorname{vol}\left(T_{1}(A, B, C)\right), \operatorname{vol}\left(T_{2}(A, B, C)\right)$ have the same representation of $\operatorname{vol}(T(A, B, C))$.

Example 4.6. For the corresponding complex analytic function $f$ of the orthoscheme formula, i.e.,

$$
\begin{array}{r}
f=\frac{1}{4}(\Lambda(A+\delta)-\Lambda(A-\delta)+\Lambda(C+\delta)-\Lambda(C-\delta) \\
\left.-\Lambda\left(\frac{\pi}{2}-B+\delta\right)+\Lambda\left(\frac{\pi}{2}-B-\delta\right)+2 \Lambda\left(\frac{\pi}{2}-\delta\right)\right)
\end{array}
$$


we have the following for case ii)

$$
\begin{aligned}
f= & \frac{1}{4}(\wedge(A+\delta)-(\wedge(A-\delta)+\pi i(A-\delta))+\wedge(C+\delta)-\wedge(C-\delta) \\
& \left.-\wedge\left(\frac{\pi}{2}-B+\delta\right)+\left(\wedge\left(\frac{\pi}{2}-B-\delta\right)+\pi i\left(\frac{\pi}{2}-B-\delta\right)\right)+2 \wedge\left(\frac{\pi}{2}-\delta\right)\right) \\
= & \frac{1}{4}\left(\wedge(A+\delta)-\wedge(A-\delta)+\wedge(C+\delta)-\wedge(C-\delta)-\wedge\left(\frac{\pi}{2}-B+\delta\right)\right. \\
& \left.\quad+\wedge\left(\frac{\pi}{2}-B-\delta\right)+2 \wedge\left(\frac{\pi}{2}-\delta\right)\right)+\frac{\pi i}{4}\left(\frac{\pi}{2}-A-B\right) \\
= & \operatorname{vol}\left(T_{1}\right)+\operatorname{vol}(I),
\end{aligned}
$$

where the most angle values remain in $(0, \pi)$ so that $\Lambda$ and $\wedge$ have the same values except for $A-\delta$ and $\frac{\pi}{2}-B-\delta$ which take values from + to - when i) changes to ii).

We have the following for case iii)

$$
\begin{aligned}
f= & \frac{1}{4}\left(\Lambda(A+\delta)-\Lambda(A-\delta)+\Lambda(C+\delta)-\Lambda(C-\delta)-\Lambda\left(\frac{\pi}{2}-B+\delta\right)\right. \\
& \left.+\Lambda\left(\frac{\pi}{2}-B-\delta\right)+2 \Lambda\left(\frac{\pi}{2}-\delta\right)\right) \\
= & \frac{1}{4}(\wedge(A+\delta)-(\wedge(A-\delta)+\pi i(A-\delta))+\wedge(C+\delta)-(\wedge(C-\delta)+\pi i(C-\delta)) \\
& \left.-\wedge\left(\frac{\pi}{2}-B+\delta\right)+\left(\wedge\left(\frac{\pi}{2}-B-\delta\right)+2 \pi i\left(\frac{\pi}{2}-B-\delta\right)\right)+2 \wedge\left(\frac{\pi}{2}-\delta\right)\right) \\
= & \operatorname{vol}\left(T_{2}\right)+\frac{\pi i}{4}\left(\frac{\pi}{2}-A-B\right)+\frac{\pi i}{4}\left(\frac{\pi}{2}-B-C\right) \\
= & \operatorname{vol}\left(T_{2}\right)+\operatorname{vol}\left(I_{1}\right)+\operatorname{vol}\left(I_{2}\right),
\end{aligned}
$$

where $A-\delta$ and $C-\delta$ change from + to - , and $\frac{\pi}{2}-B-\delta$ changes $+\rightarrow-\rightarrow+$ when i) $\rightarrow$ ii $\rightarrow$ iii), other values are remaining at $(0, \pi)$.

Now we consider a more general situation than that of Theorem 4.3. We take a Lambert cube $L C$ with dihedral angles $A, B, C$, and then we extend the four faces of the Lambert cube. Finally, we obtain a tetrahedron in the extended hyperbolic space (see Fig. 11). Then edge $\langle 14\rangle$ is lying outside $\partial H^{3}$, so the dihedral angle will have a negative pure imaginary value with respect to the (clockwise contour) definition of the dihedral angle (see Definitions 3.3, 3.6 and Fig. 12 in [3]). The value $b$ is the distance between two faces $\langle 2589\rangle$ and $\langle 36710\rangle$ and is the same as the length of edge $\langle 78\rangle$.

Now let us compare the analytic function $f$ (for the volume of a hyperbolic orthoscheme) and the volume integration (for the orthoscheme whose one edge $\langle 14\rangle$ is lying outside $\left.\partial H^{3}\right)$ in the extended hyperbolic space.

Example 4.7. From the orthoscheme formula $f$ in Example 4.6, we get the orthoscheme analytic function with three dihedral angles $A,-i b, C$.

$$
f(A,-i b, C)=\frac{1}{4}(\Lambda(A+\delta)-\Lambda(A-\delta)+\Lambda(C+\delta)-\Lambda(C-\delta)
$$




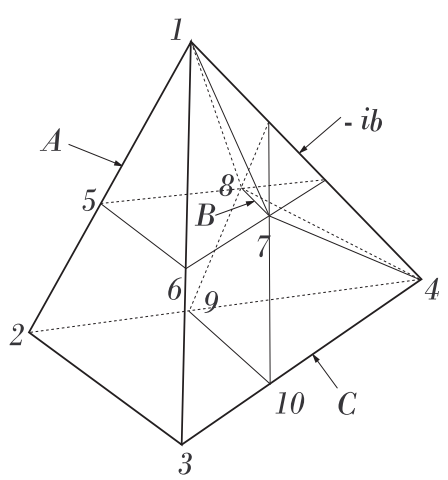

Figure 11

$$
\left.-\Lambda\left(\frac{\pi}{2}+i b+\delta\right)+\Lambda_{\gamma}\left(\frac{\pi}{2}+i b-\delta\right)+2 \Lambda\left(\frac{\pi}{2}-\delta\right)\right),
$$

where $\delta=\tan ^{-1} \frac{\sqrt{\cosh ^{2} b-\sin ^{2} A \sin ^{2} C}}{\cos A \cos C}$.

By observation of the contour chasing in Example 4.6, we have to replace (see Fig. 12)

$$
\begin{aligned}
\Lambda(A-\delta) & \rightarrow \wedge(A-\delta)+\pi i(A-\delta), \\
\Lambda(C-\delta) & \rightarrow \wedge(C-\delta)+\pi i(C-\delta), \\
\Lambda_{\gamma}\left(\frac{\pi}{2}+i b-\delta\right) & \rightarrow \Lambda\left(\frac{\pi}{2}+i b-\delta\right)+2 \pi i\left(\frac{\pi}{2}+i b-\delta\right),
\end{aligned}
$$

so we obtain

$$
\begin{aligned}
f= & \frac{1}{4}\left(\wedge(A+\delta)-\wedge(A-\delta)+\wedge(C+\delta)-\wedge(C-\delta)-\Lambda\left(\frac{\pi}{2}+i b+\delta\right)\right. \\
& \left.+\Lambda\left(\frac{\pi}{2}+i b-\delta\right)+2 \wedge\left(\frac{\pi}{2}-\delta\right)\right)+\frac{\pi i}{4}(\pi-A-C+2 i b) . \\
\qquad \underbrace{\uparrow}_{\gamma} \longrightarrow & \uparrow
\end{aligned}
$$
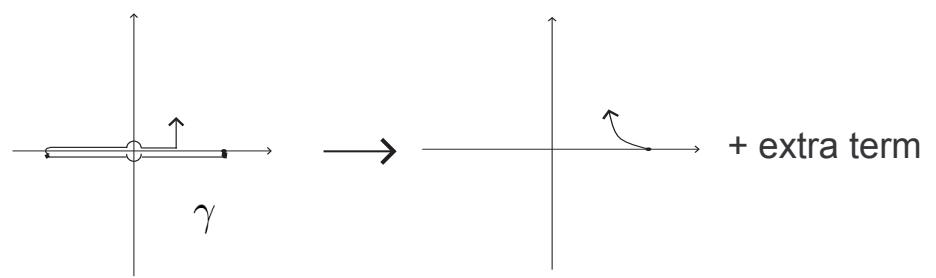

FiguRE 12

Now let us calculate the integral volume of the tetrahedron $\langle 1234\rangle$ in the extended hyperbolic space. We know easily that the polyhedra $\langle 15678\rangle$ and 
$\langle 478910\rangle$ are the half of lune with quadrangle section, and the tetrahedron $\langle 1478\rangle$ has two opposite dihedral angles $-i b, \pi-B$ and other rectangular dihedral angles.

We know that

$$
\begin{aligned}
\operatorname{vol}\langle 15678\rangle & =\frac{\pi i}{4}\left(2 \pi-\frac{\pi}{2}-\frac{\pi}{2}-\frac{\pi}{2}-A\right)=\frac{\pi i}{4}\left(\frac{\pi}{2}-A\right), \\
\operatorname{vol}\langle 478910\rangle & =\frac{\pi i}{4}\left(\frac{\pi}{2}-C\right), \\
\operatorname{vol}\langle 1478\rangle & =-\frac{i}{2}(\pi-B)(-i b)=\frac{b(B-\pi)}{2} .
\end{aligned}
$$

Note that the last formula can be obtained by the symmetric property of the extended hyperbolic space. A tetrahedron with two opposite dihedral angles $A, B$ and other rectangular dihedral angles has a volume of $\frac{A}{2 \pi} \times \frac{B}{2 \pi} \times \operatorname{vol}\left(\mathbb{S}_{H}^{3}\right)=$ $-\frac{i}{2} A B$ (spherical case, $\frac{A}{2 \pi} \times \frac{B}{2 \pi} \times \operatorname{vol}\left(\mathbb{S}^{3}\right)=\frac{1}{2} A B$ ). By direct integration, we can also obtain the same result.

$$
\begin{aligned}
\lim _{\epsilon \rightarrow 0} \int_{\langle 1478\rangle} \frac{d x \wedge d y \wedge d z}{\left(d_{\epsilon}^{2}-\left(x^{2}+y^{2}+z^{2}\right)\right)^{2}} & =\lim _{\epsilon \rightarrow 0} \int_{0}^{z_{0}} \int_{0}^{\alpha} \int_{0}^{\infty} \frac{r}{\left(d_{\epsilon}^{2}-\left(r^{2}+z^{2}\right)\right)^{2}} d r d \theta d z \\
& =\lim _{\epsilon \rightarrow 0} \frac{\alpha}{2} \int_{0}^{z_{0}} \frac{d z}{z^{2}-d_{\epsilon}^{2}} \\
& =-\frac{b \alpha}{2}
\end{aligned}
$$

where we locate the edge $\langle 78\rangle$ on interval $\left[0, z_{0}\right]$ of the $z$-coordinate, and $z_{0}=$ $\tanh b$ is the $z$-coordinate value corresponding to the edge length $b$, and $\alpha=$ $\pi-B$.

We know the volume of a Lambert cube $L C(A, B, C)$, i.e., the polyhedron $\langle 235678910\rangle$, by Kellerhals result [8].

$$
\begin{aligned}
\operatorname{vol}(L C)= & \frac{1}{4}(\wedge(A+\delta)-\wedge(A-\delta)+\wedge(B+\delta)-\wedge(B-\delta)+\wedge(C+\delta) \\
& \left.-\wedge(C-\delta)+4 \wedge\left(\frac{\pi}{2}-\delta\right)-2 \wedge(\delta)\right),
\end{aligned}
$$

where $\delta=\tan ^{-1} \frac{\sqrt{\cosh ^{2} b-\sin ^{2} A \sin ^{2} C}}{\cos A \cos C}$.

Hence the volume of the tetrahedron $\langle 1234\rangle$ can be obtained by summing up the volumes of the four polyhedra, so we get finally

$$
\begin{aligned}
& 4(\operatorname{vol}\langle 1234\rangle-f(A,-b i, C)) \\
= & 2 b B+\Lambda\left(\frac{\pi}{2}+i b+\delta\right)-\Lambda\left(\frac{\pi}{2}+i b-\delta\right)+\wedge(\delta+B)+\wedge(\delta-B)-\wedge \\
= & : g(b, B),
\end{aligned}
$$

where $\tan B=\tanh b \tan \delta$. We know that $\sin (\delta+B) \sin (\delta-B)=\sin ^{2} \delta-\sin ^{2} B$, $\cos (i b+\delta) \cos (i b-\delta)=\cos ^{2} \delta+\sinh ^{2} b, \sinh ^{2} b=\frac{\cos ^{2} \delta \sin ^{2} B}{\sin ^{2} \delta-\sin ^{2} B}$, and $\tan B=$ 
$\tanh b \tan \delta \Leftrightarrow 2 b=\log \frac{\sin (\delta+B)}{\sin (\delta-B)}$. Hence, we get $g(0,0)=0$ and

$$
\begin{gathered}
\frac{\partial g}{\partial B}=-\delta_{B} \log \frac{\sin (\delta+B) \sin (\delta-B) \sin \left(\frac{\pi}{2}+i b+\delta\right) \sin \left(\frac{\pi}{2}+i b-\delta\right)}{\sin ^{2} \delta \cos ^{2} \delta}+2 b+\log \frac{\sin (\delta-B)}{\sin (\delta+B)}=0, \\
\frac{\partial g}{\partial b}=-\delta_{b} \log \frac{\sin (\delta+B) \sin (\delta-B) \sin \left(\frac{\pi}{2}+i b+\delta\right) \sin \left(\frac{\pi}{2}+i b-\delta\right)}{\sin ^{2} \delta \cos ^{2} \delta}+2 B+i \log \frac{\cos (i b-\delta)}{\cos (i b+\delta)}=0 .
\end{gathered}
$$

Finally, we get $\operatorname{vol}\langle 1234\rangle=f(A,-b i, C)$ for clockwise contour chasing, and conclude that the analytic orthoscheme formula gives the volume of the generalized orthoscheme on $\mathbb{S}_{H}^{3}$ while permitting the one-edge outside condition.

Furthermore, we get the same conclusion for counterclockwise contour chasing. For the counterclockwise version of the angle definition, we have to change $-i b \rightarrow i b, f(A,-i b, C) \rightarrow f(A, i b, C)$, for the counterclockwise contour chasing of the analytic volume function, we have to change $\wedge(A-\delta)+\pi i(A-$ $\delta) \rightarrow \wedge(A-\delta)-\pi i(A-\delta), \wedge(C-\delta)+\pi i(C-\delta) \rightarrow \wedge(C-\delta)-\pi i(C-\delta)$, $\Lambda_{\gamma}\left(\frac{\pi}{2}+i b-\delta\right)=\Lambda\left(\frac{\pi}{2}+i b-\delta\right)+2 \pi i\left(\frac{\pi}{2}+i b-\delta\right) \rightarrow \Lambda_{\bar{\gamma}}\left(\frac{\pi}{2}-i b-\delta\right)=\Lambda\left(\frac{\pi}{2}-i b-\delta\right)-$ $2 \pi i\left(\frac{\pi}{2}-i b-\delta\right)$, and from the counterclockwise volume definition of the extended hyperbolic space, we have to change vol $\langle 15678\rangle=\frac{\pi i}{4}\left(\frac{\pi}{2}-A\right) \rightarrow-\frac{\pi i}{4}\left(\frac{\pi}{2}-A\right)$, $\operatorname{vol}\langle 478910\rangle=\frac{\pi i}{4}\left(\frac{\pi}{2}-C\right) \rightarrow-\frac{\pi i}{4}\left(\frac{\pi}{2}-C\right), \operatorname{vol}\langle 1478\rangle=-\frac{i}{2}(\pi-B)(-i b)=$ $\frac{b(B-\pi)}{2} \rightarrow \frac{i}{2}(\pi-B)(i b)=\frac{b(B-\pi)}{2}$. Hence, we get

$$
\begin{aligned}
& 4(\operatorname{vol}\langle 1234\rangle-f(A, b i, C)) \\
= & 2 b B+\Lambda\left(\frac{\pi}{2}-i b+\delta\right)-\Lambda\left(\frac{\pi}{2}-i b-\delta\right)+\wedge(\delta+B)+\wedge(\delta-B)-\wedge \\
= & k(b, B),
\end{aligned}
$$

where $\tan B=\tanh b \tan \delta$.

Similarly, we conclude $k(b, B)=0$ and $\operatorname{vol}\langle 1234\rangle=f(A, b i, C)$ for counterclockwise contour chasing.

From the above argument, we summarize the results for clockwise(-) or counterclockwise $(+)$ contour chasing as follows:

$$
\begin{aligned}
f(A, \mp b i, C) & =\operatorname{vol}\langle 1234\rangle \\
& =\operatorname{vol}(L C(A, B, C))+\operatorname{vol}\langle 15678\rangle+\operatorname{vol}\langle 478910\rangle+\operatorname{vol}\langle 1478\rangle,
\end{aligned}
$$

and

$$
\begin{aligned}
& \operatorname{Re} f(A, \mp b i, C)=\operatorname{vol}(L C(A, B, C))+\operatorname{vol}\langle 1478\rangle, \\
& \operatorname{Im} f(A, \mp b i, C)=\operatorname{vol}\langle 15678\rangle+\operatorname{vol}\langle 478910\rangle .
\end{aligned}
$$

Note that the formula $\operatorname{Re} f(A,-b i, C)$ in $(17)$ is the same as one choice among multi-values of Theorem 1 in [9]. In [9], Authors used $a_{k}=e^{i \theta_{k}}$ and $a_{4}=e^{l}$, so they implicitly adopted the clockwise angle definition.

From now on we assume tetrahedra whose edges can be placed outside $\mathbb{H}^{3}$, but whose faces cannot be placed outside $\mathbb{H}^{3}$. Then we can divide a tetrahedron into orthoschemes of types i), ii), iii) and iv). 


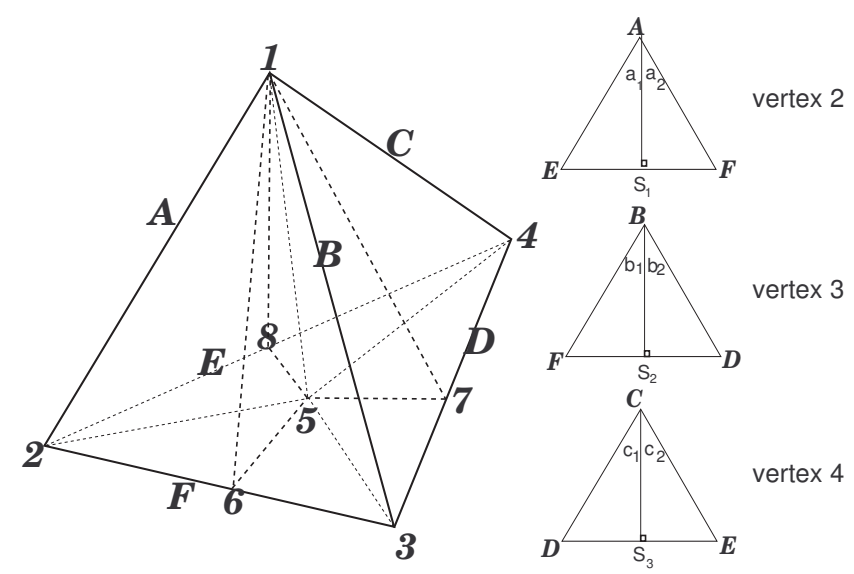

FiguRe 13
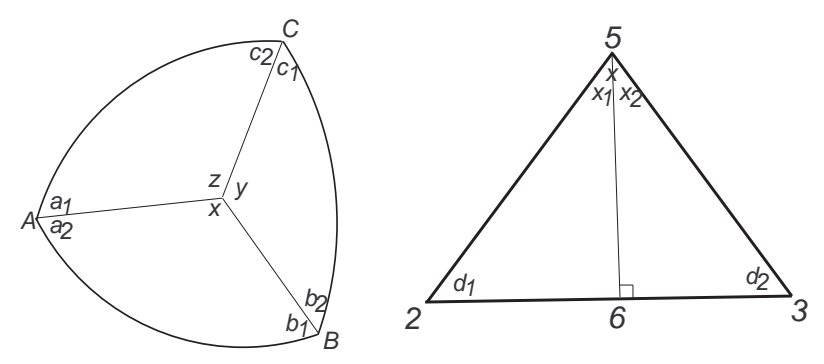

FIGURE 14

First, we draw a perpendicular from vertex 1 to the face $\langle 234\rangle$ and assume that the perpendicular foot 5 is lying on $\langle 234\rangle \cap \mathbb{H}^{3}$, then we get 3 tetrahedra with 9 new dihedral angle variables: $a_{1}, a_{2}, b_{1}, b_{2}, c_{1}, c_{2}$ and $x, y, z$. At vertices $2,3,4$, we can obtain $a_{i}, b_{i}, c_{i}$ from the perpendicularity condition (see Fig. 13). If vertex 2 is a hyperbolic (or Lorentzian) point, then we obtain a spherical (or hyperbolic) triangle $(A, E, F)$ obtained by a dual plane $2^{\perp}$ and the tetrahedron $\langle 1234\rangle$. The vertices of the hyperbolic triangle $(A, E, F)$ could be located outside $\mathbb{H}^{3}$, so we need general trigonometry. Note that the trigonometry of general position works well (see [3]), and is the same as standard trigonometry.

Therefore, we easily get

$$
\frac{\cos E}{\sin a_{1}}=\frac{\cos F}{\sin a_{2}}
$$


for any (spherical or hyperbolic) type of triangle $(A, E, F)$. From (18) and $a_{2}=A-a_{1}$, we obtain

$$
a_{1}=\cot ^{-1}\left(\frac{\cos A \cos E+\cos F}{\sin A \cos E}\right),
$$

and the formula (19) also works well for the case that the vertex 2 is an ideal point. We know $\cot ^{-1} z=\frac{1}{2 i} \log \frac{z+i}{z-i}$, so $A=0$ or $E=\frac{\pi}{2}$ implies $a_{1}=0$ and does not disturb the analyticity of $a_{1}$. We also get similar results for $b_{1}, b_{2}, c_{1}, c_{2}$.

Second, if vertex 1 is a hyperbolic (or Lorentzian) point, then we also obtain a spherical (or hyperbolic) triangle $(A, B, C)$ (see Fig. 14). So we have $\frac{\cos A \cos B+\cos C}{\sin A \sin B}=\frac{\cos a_{2} \cos b_{1}+\cos x}{\sin a_{2} \sin b_{1}}$ for any (spherical or hyperbolic) type of triangle $(A, B, C)$, hence $x=\cos ^{-1}\left(\frac{\sin a_{2} \sin b_{1}}{\sin A \sin B}(\cos A \cos B+\cos C)-\cos a_{2} \cos b_{1}\right)$, it works as well for an Euclidean triangle case, i.e., vertex 1 is an ideal point. We know that $\cos ^{-1} z=-i \log \left(z+\left(z^{2}-1\right)^{\frac{1}{2}}\right)$.

In this paper, we consider only non-degenerate tetrahedra. An ordinary hyperbolic tetrahedron does not have a 0 dihedral angle. However, in our consideration of non-degenerate tetrahedra, a 0 dihedral angle is possible, when an edge of the tetrahedron is tangent to $\partial \mathbb{H}^{3}$. From formula (19), $\frac{\sin a_{1}}{\sin A}=$ $\frac{\cos a_{1} \cos E}{\cos A \cos E+\cos F} \rightarrow \frac{\cos E}{\cos E+\cos F}$, whenever $A \rightarrow 0$ (then $a_{i} \rightarrow 0$ ). Also from formula (18), we get $\frac{\sin a_{2}}{\sin A} \rightarrow \frac{\cos F}{\cos E+\cos F}$, whenever $A \rightarrow 0$. Therefore the analyticity of the dihedral angles of $x, y, z$ is not a problem, even for the edgetangent case.

Third, if we draw a perpendicular from 5 to the edge $\langle 23\rangle$ and denote the foot of the perpendicular as 6 , then we obtain the additional line $\langle 16\rangle$ perpendicular to the edge $\langle 23\rangle$. By considering some isometric transformation that sends the triangle $\langle 234\rangle$, the line $\langle 15\rangle$, and the point 5 to the $y z$-plane, $z$-axis, and center 0 of the Kleinian model, respectively, we get the desired perpendicularity property. Then the angles $d_{1}, d_{2}$ in the generalized triangle $\langle 235\rangle$ are obtained as the spherical length of $S_{1} F$ and $F S_{2}$ (see Figs. 13 and 14), so we have $d_{1}=\cos ^{-1}\left(\frac{\cos a_{2}}{\sin F}\right), d_{2}=\cos ^{-1}\left(\frac{\cos b_{1}}{\sin F}\right)$. Also, changing $A \rightarrow x$, $a_{1} \rightarrow x_{1}, E \rightarrow d_{1}$, and $F \rightarrow d_{2}$ at formula (19), we know the value $x_{1}$ and $x_{2}=x-x_{1}$. Hence we can find an analytic expression of $a_{i}, b_{i}$, and $x_{i}$ with variables the original dihedral angles $A, \ldots, F$. Therefore we deduce two orthoschemes $T\left(x_{1}, a_{2}, F\right)$ and $T\left(x_{2}, b_{1}, F\right)$ with analytic expressions from the tetrahedron $\langle 1235\rangle$. Similarly, we can also divide the tetrahedra $\langle 1345\rangle$ and $\langle 1245\rangle$ individually into two orthoschemes, so we get the following relations:

$$
\begin{aligned}
\operatorname{vol}\langle 1234\rangle & =\operatorname{vol}\langle 1256\rangle+\operatorname{vol}\langle 1356\rangle+\cdots+\operatorname{vol}\langle 1258\rangle \\
& =f\left(x_{1}, a_{2}, F\right)+f\left(x_{2}, b_{1}, F\right)+\cdots+f\left(z_{2}, a_{1}, E\right) \\
& =f(A, B, C, D, E, F),
\end{aligned}
$$


where the second equality is obtained by Examples 4.6 and 4.7, and the third equality by the coincidence of two analytic functions at the usual hyperbolic tetrahedron and an extension of the same kind of contour chase.

Additionally, we have to consider the case in which vertex 5 is located outside the triangle $\langle 234\rangle$ and is lying inside or outside of $\mathbb{H}^{3}$. The inside case (simply denote as " $5 \in \mathbb{H}^{3}$ ") will arise, if some dihedral angles of the tetrahedron $\langle 1234\rangle$ are obtuse. If foot 5 of the perpendicular emanating from vertex 1 is lying outside of the triangle $\langle 234\rangle$, then the original tetrahedron is easily obtained by union of two tetrahedra minus one tetrahedron with a common perpendicular edge or one tetrahedra minus two tetrahedra with a common perpendicular edge, so we can solve the obtuse problem. Actually, we have to explain analyticity at each divided dihedral angle, however we will omit this, since the method is similar to the comparison technique described around Fig. 8. Furthermore, we have to consider the remaining case $5 \notin \mathbb{H}^{3}$ : If one dihedral angle is not 0 , then we can apply the same dividing method described in Fig. 8 and we can obtain one hyperbolic vertex for each divided piece, so we can transform this case to $5 \in \mathbb{H}^{3}$ case, and if all dihedral angles are 0 , then the tetrahedron becomes a regular tetrahedron and the case becomes $5 \in \mathbb{H}^{3}$ case. Hence we finish the checking of the two cases: $5 \in \mathbb{H}^{3}$ case and $5 \notin \mathbb{H}^{3}$ case.

In order to complete the proof of the following theorem, we have to check the analyticity of the orthoscheme volume function $f(A, B, C)$ from iii) to iv) of Fig. 10. From the well-known dihedral angle condition of an orthoscheme and Lambert cube, the value inside of $\Lambda(\cdot)$ does not touch the singular point $0, \pi$, so we need to examine only the singular case $B=0$ between iii) and iv), at which the edge $\langle 14\rangle$ is tangent to the ideal boundary $\partial \mathbb{H}^{3}$. If the analytic function $\Lambda(\cdot)$ of $f(A, 0, C)$ touches the singular position, i.e., $A, C= \pm \delta$ or $\pi \pm \delta$, then $A=\frac{\pi}{2}$ or $C=\frac{\pi}{2}$ are easily obtained. Then the orthoschemes $f\left(\frac{\pi}{2}, 0, C\right)$ and $f\left(A, 0, \frac{\pi}{2}\right)$ (for example, whose four vertices are $(0,0,0),(p, 0,0),(0, \infty, 0)$, and $(0,0,1)$ in the extended Kleinian model) become the case of one vertex lying on the ideal boundary. That is a situation where both the vertex ideal and edge tangent cases arise, simultaneously. Furthermore, we must consider the degenerate (i.e., flat) orthoscheme $f\left(\frac{\pi}{2}, 0, \frac{\pi}{2}\right)$, which becomes a non-singular case by easy calculation. Therefore, the genuine edge-tangent case without the vertex ideal condition is not a singular but an analytic case.

Therefore, we conclude the following theorem.

Theorem 4.8. For a hyperbolic tetrahedron with six dihedral angle variables, if we move vertices out continuously from inside to outside of $\mathbb{H}^{3}$ keeping every face of the tetrahedron intersecting the hyperbolic space $\mathbb{H}^{3}$, then the volume formula is expressed by the same analytic multi-valued function. This analytic function takes a unique value for a clockwise (counterclockwise resp.) contour choice, and this choice coincides with the volume of the tetrahedron of the fixed extended hyperbolic space defined from the clockwise (counterclockwise resp.) 
contour. When the moving vertex is on the ideal boundary $\partial \mathbb{H}^{3}$, the volume function is not analytic but is continuous at the point. In particular, at the edge-tangent case (i.e., when the dihedral angle at the edge is 0 ) the volume function of dihedral angle variables is analytic.

Now we examine the volume of a regular tetrahedron under the face-intersecting condition.

Example 4.9. From example 1 in [4], we know the volume formula of a regular tetrahedron with dihedral angle $A$, so we can obtain the analytic function $f(A)$ of the regular tetrahedron.

$$
\begin{aligned}
f(A)= & 2 \Lambda\left(\frac{A+w}{2}\right)-2 \Lambda\left(\frac{A-w}{2}\right)+\Lambda\left(\frac{\pi-2 A-w}{2}\right)+\Lambda\left(\frac{\pi+2 A-w}{2}\right) \\
& -\Lambda\left(\frac{\pi+w}{2}\right)+\Lambda\left(\frac{\pi-w}{2}\right),
\end{aligned}
$$

where $w=\cos ^{-1}\left(\frac{1-\cos A}{2 \cos A}\right)$.

First, we consider the real dihedral angle case. The dihedral angle $A$ takes its value from the interval $\left[0, \cos ^{-1} \frac{1}{3}\right.$ ), more precisely $A=\cos ^{-1} \frac{1}{3}$ (Euclidean tetrahedron, i.e., one point in hyperbolic space) $\rightarrow A=\frac{\pi}{3}$ (4 ideal vertices case) $\rightarrow A=0$ (hyperideal, i.e., all edges tangent to $\partial \mathbb{H}^{3}$ ).

We can check that $w, \frac{A-w}{2}$, and $\frac{\pi-2 A-w}{2}$ move along $0 \rightarrow \frac{\pi}{3} \rightarrow \frac{\pi}{2},+\rightarrow 0 \rightarrow$ - , and $+\rightarrow 0 \rightarrow+$, respectively. Only 2 terms $\frac{A-w}{2}$ and $\frac{\pi-2 A-w}{2}$ touch the singular value. Hence, we can obtain

$$
\begin{aligned}
\Lambda\left(\frac{A-w}{2}\right) & =\wedge\left(\frac{A-w}{2}\right)+\pi i\left(\frac{A-w}{2}\right), \\
\Lambda\left(\frac{\pi-2 A-w}{2}\right) & =\wedge\left(\frac{\pi-2 A-w}{2}\right)+2 \pi i\left(\frac{\pi-2 A-w}{2}\right),
\end{aligned}
$$

and finally, we get the following formula for $A \in\left[0, \frac{\pi}{3}\right]$ :

$$
\begin{aligned}
f(A)= & 2 \wedge\left(\frac{A+w}{2}\right)-2 \wedge\left(\frac{A-w}{2}\right)+\wedge\left(\frac{\pi-2 A-w}{2}\right)+\wedge\left(\frac{\pi+2 A-w}{2}\right) \\
& -\wedge\left(\frac{\pi+w}{2}\right)+\wedge\left(\frac{\pi-w}{2}\right)+\pi i(\pi-3 A) \\
= & \operatorname{Re} f(A)+\pi i(\pi-3 A) .
\end{aligned}
$$

Note that the Lobachevsky function $\wedge(\cdot)$ always takes real values. Also, we already know the following formula for $A \in\left[\frac{\pi}{3}, \cos ^{-1} \frac{1}{3}\right]$ :

$$
\begin{aligned}
f(A)= & 2 \wedge\left(\frac{A+w}{2}\right)-2 \wedge\left(\frac{A-w}{2}\right)+\wedge\left(\frac{\pi-2 A-w}{2}\right)+\wedge\left(\frac{\pi+2 A-w}{2}\right) \\
& -\wedge\left(\frac{\pi+w}{2}\right)+\wedge\left(\frac{\pi-w}{2}\right) \\
= & \operatorname{Re} f(A) .
\end{aligned}
$$

For the case $A=0$, every edge is tangent to the ideal boundary $\partial \mathbb{H}^{3}$ and hence the tetrahedron is hyperideal. If we truncate 4 tetrahedra for each vertex 
with its dual plane, then the remaining tetrahedron is a regular ideal octahedron. All dihedral angles of the octahedron are $\frac{\pi}{2}$, so the tetrahedron with all 0 dihedral angles has a volume of

$$
\operatorname{vol}(\text { regular ideal octahedron })+4 \times \frac{\pi i}{4}(\pi-0-0-0)=8 \wedge\left(\frac{\pi}{4}\right)+\pi^{2} i .
$$

In fact, the volume of the 0 dihedral angle tetrahedron is also obtained from the above analytic function $f(A)$.

$$
\begin{aligned}
f(0)= & 2 \wedge\left(\frac{w}{2}\right)-2 \wedge\left(\frac{-w}{2}\right)+\wedge\left(\frac{\pi-w}{2}\right)+\wedge\left(\frac{\pi-w}{2}\right)-\wedge\left(\frac{\pi+w}{2}\right)+\wedge\left(\frac{\pi-w}{2}\right) \\
& +\pi^{2} i \\
= & 8 \wedge\left(\frac{\pi}{4}\right)+\pi^{2} i
\end{aligned}
$$

where $w=\cos ^{-1} 0=\frac{\pi}{2}$. Note that, for $A=0$, the inside 6 values of $\wedge(\cdot)$ become $\pm \frac{\pi}{4}, \frac{3 \pi}{4}$, so they never touch the singular value $0, \pi$, hence we can check the analyticity of the edge tangent case one more time.

Second, we consider the pure imaginary dihedral angle case. The dihedral angle $A$ takes values from the interval $[0,-\infty i)$, more precisely $A=0$ (hyperideal case) $\rightarrow A=-i b \rightarrow A=-\infty i$, and then $w$ moves along $\frac{\pi}{2} \rightarrow \frac{2 \pi}{3}$. Only 2 terms $\frac{-i b-w}{2}, \frac{\pi+2 i b-w}{2}$ still have to be considered; the value $\frac{-i b-w}{2}$ does not cross the branch cut of $\Lambda(z)$, since the values moves along $+\rightarrow$ clockwise around $0 \rightarrow-\rightarrow \frac{-i b-w}{2}$. By the contour chasing $\left(\gamma^{\prime}\right)$ of $\frac{\pi+2 i b-w}{2}$, we have the following relation similar to Example 4.7 and Fig. 12:

$$
\Lambda_{\gamma^{\prime}}\left(\frac{\pi+2 i b-w}{2}\right)=\Lambda\left(\frac{\pi+2 i b-w}{2}\right)+2 \pi i\left(\frac{\pi+2 i b-w}{2}\right) .
$$

Hence we get (clockwise approach)

$$
\begin{aligned}
f(-i b)= & 2 \Lambda\left(\frac{-i b+w}{2}\right)-2 \Lambda\left(\frac{-i b-w}{2}\right)+\Lambda\left(\frac{\pi+2 i b-w}{2}\right)+\Lambda\left(\frac{\pi-2 i b-w}{2}\right) \\
& -\wedge\left(\frac{\pi+w}{2}\right)+\wedge\left(\frac{\pi-w}{2}\right)+\pi i(\pi+2 i b-w), w=\cos ^{-1}\left(\frac{1-\cosh b}{2 \cosh b}\right) .
\end{aligned}
$$

A regular tetrahedron with dihedral angle $-i b$ is divided into 11 pieces of a polyhedron by a dividing similar to that in the Lambert cube case in the extended hyperbolic space (see Fig. 15).

From Theorem 4.8, we know $f(-i b)=\operatorname{vol}\langle 1234\rangle=\operatorname{vol}(a)+4 \times \operatorname{vol}(b)+6 \times$ $\operatorname{vol}(c)$, and $\operatorname{vol}(b)=\frac{\pi i}{4}(4 \pi-3 \pi)=\frac{\pi^{2}}{4} i, \operatorname{vol}(c)=-\frac{i}{2}(-i b)(\pi-\alpha)=-\frac{b}{2}(\pi-$ $\alpha), \operatorname{vol}(a)=\operatorname{Re} f(\alpha)$. Here we can derive the relation between $b$ and $\alpha$, by generalized trigonometry (see [3]) in the extended hyperbolic space for a dual triangle by a polar dual truncation at vertex 1 and the face triangle $\langle 123\rangle$ (see Fig. 16). Then we obtain $\cos \theta=\frac{\cosh ^{2} b+\cosh b}{\sinh ^{2} b}=\frac{\cos ^{2} \alpha+\cos \alpha}{\sin ^{2} \alpha}$, and simply $\cos \alpha=\frac{\cosh b}{2 \cosh b-1}$ and $\cosh b=\frac{\cos \alpha}{2 \cos \alpha-1}$. 


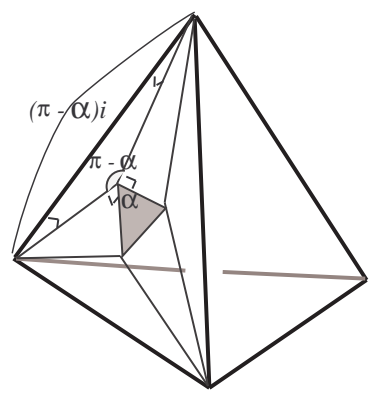

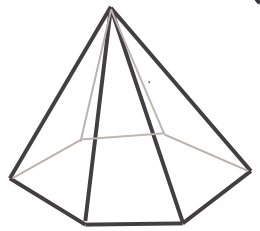

(b)

FiguRE 15
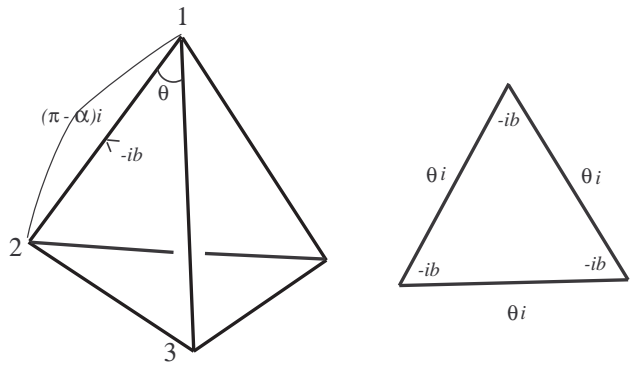

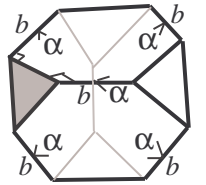

(a)

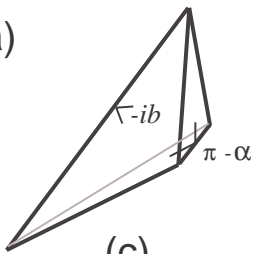

(c)

FiguRE 16

Therefore, we get

$$
\begin{aligned}
& \operatorname{Re} f(-i b)=\operatorname{Re} f(\alpha)-3 b(\pi-\alpha), \\
& \operatorname{Im} f(-i b)=\pi^{2} i, \quad \cos \alpha=\frac{\cosh b}{2 \cosh b-1} .
\end{aligned}
$$

If we apply the following formula (which comes from (3)) to (20), we can calculate the exact value of $f(-i b)$.

$$
\Lambda\left(\frac{\pi}{2}-\delta+i b\right)-\Lambda\left(\frac{\pi}{2}+\delta+i b\right)=\sum_{n=1}^{\infty} \frac{(-1)^{n+1} e^{-2 n b}}{n^{2}} \sin 2 n \delta+2 \delta b, \quad \delta, b>0 .
$$

Our method can be used to obtain the volume of a more general 4-valent regular ideal polyhedron from some hyperideal polyhedron.

As one can easily imagine, in fact, we strongly believe that the analysis we had in this paper can be extended to every type of tetrahedron in extended 
hyperbolic space. A complete investigation of the analyticity and branch problem of the volume formula for a tetrahedron in extended hyperbolic space will be deferred to a subsequent paper.

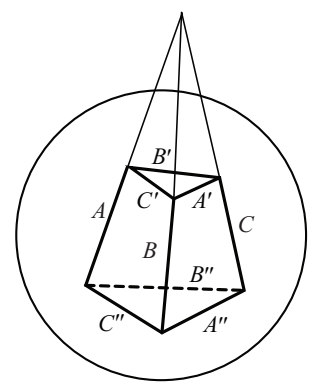

(a)

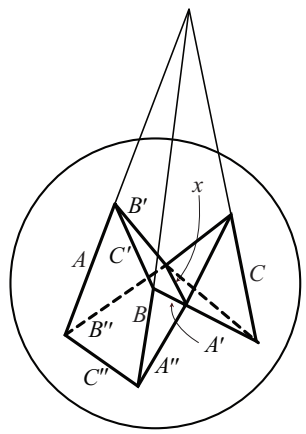

(b)

FIGURE 17

Now we introduce some elementary applications of our theorem. Suppose we have a hyperbolic prism $P$ with 9 dihedral angles (see Fig. 17). Then $P$ determines a unique trihedron obtained by extending the three faces. By considering two upper (lower resp.) tetrahedra in this trihedron, we can easily derive the following first (second resp.) relation about the volume of prism $P\left(A, B, C, A^{\prime}, B^{\prime}, C^{\prime}, A^{\prime \prime}, B^{\prime \prime}, C^{\prime \prime}\right)$ by Theorem 4.1 :

volume of prism

$$
\begin{aligned}
& =f\left(A, B, C, A^{\prime \prime}, B^{\prime \prime}, C^{\prime \prime}\right)-f\left(A, B, C, \pi-A^{\prime}, \pi-B^{\prime}, \pi-C^{\prime}\right) \\
& =f\left(A, B, C, A^{\prime}, B^{\prime}, C^{\prime}\right)-f\left(A, B, C, \pi-A^{\prime \prime}, \pi-B^{\prime \prime}, \pi-C^{\prime \prime}\right) .
\end{aligned}
$$

Note that

$$
\begin{aligned}
& \frac{\pi i}{2}(\pi-A-B-C) \\
= & f\left(A, B, C, A^{\prime}, B^{\prime}, C^{\prime}\right)+f\left(A, B, C, \pi-A^{\prime}, \pi-B^{\prime}, \pi-C^{\prime}\right) \\
= & f\left(A, B, C, A^{\prime \prime}, B^{\prime \prime}, C^{\prime \prime}\right)+f\left(A, B, C, \pi-A^{\prime \prime}, \pi-B^{\prime \prime}, \pi-C^{\prime \prime}\right) .
\end{aligned}
$$

The above relation (22) can also be applied to other types of volume relation. For example, if the upper and lower triangles in Fig. 17(a) intersect each other, then we can obtain one prism and one tetrahedron (see Fig. 17(b)). Hence, we can see the following relation:

$$
\begin{aligned}
& P\left(A, B, C, A^{\prime}, B^{\prime}, C^{\prime}, A^{\prime \prime}, B^{\prime \prime}, C^{\prime \prime}\right) \\
= & f\left(A, B, C, A^{\prime \prime}, B^{\prime \prime}, C^{\prime \prime}\right)-f\left(A, B, C, \pi-A^{\prime}, \pi-B^{\prime}, \pi-C^{\prime}\right) \\
= & P\left(x, C^{\prime}, C^{\prime \prime}, A, B^{\prime \prime}, B^{\prime}, B, A^{\prime \prime}, A^{\prime}\right)-f\left(\pi-A^{\prime \prime}, \pi-B^{\prime \prime}, C, \pi-B^{\prime}, \pi-A^{\prime}, x\right),
\end{aligned}
$$

where the dihedral angle $x$ is obtained from the Gram matrix of the prism. 
One can also easily and naturally derive some other relations for simple hyperbolic polyhedra without a dividing process. We expect that extended hyperbolic space will provide a nice method of demonstrating the analyticity of the volume of a given combinatorial type (convex) hyperbolic polyhedron.

\section{Appendix}

Now we supply another elementary proof of Theorem 3.2. In this proof, we use only elementary geometric properties of the extended hyperbolic space, without using the Ushijima's result.

We know the volume formula (see (6)) for a hyperbolic tetrahedron with three vertices at infinity and one hyperbolic vertex using Vinberg's geometric technique (see [1] or [14]). Similarly, we derive and show the volume formula for a hyperbolic tetrahedron with three vertices at infinity and one Lorentzian vertex below.

First, we consider a tetrahedron $S$ with three ideal points 1, 2, 3 on one ideal boundary and one Lorentzian point 0 (see Fig. 18), and we extend the lines $\langle 10\rangle,\langle 20\rangle,\langle 30\rangle$ to the other ideal boundary (which is different from the first one) points and denote them as $1^{\prime}, 2^{\prime}, 3^{\prime}$, respectively. Then we can construct an octahedron with eight faces $\langle 123\rangle,\left\langle 1^{\prime} 23\right\rangle,\left\langle 12^{\prime} 3\right\rangle,\left\langle 123^{\prime}\right\rangle,\left\langle 1^{\prime} 2^{\prime} 3\right\rangle,\left\langle 1^{\prime} 23^{\prime}\right\rangle,\left\langle 12^{\prime} 3^{\prime}\right\rangle$, $\left\langle 1^{\prime} 2^{\prime} 3^{\prime}\right\rangle$.

If one ideal boundary has $m$ ideal vertices of a given ideal polyhedron and the other ideal boundary has its remaining $n$ ideal vertices, then we simply call it an $(m, n)$ ideal polyhedron. Hence, the usual ideal tetrahedron is a $(4,0)$ ideal tetrahedron.

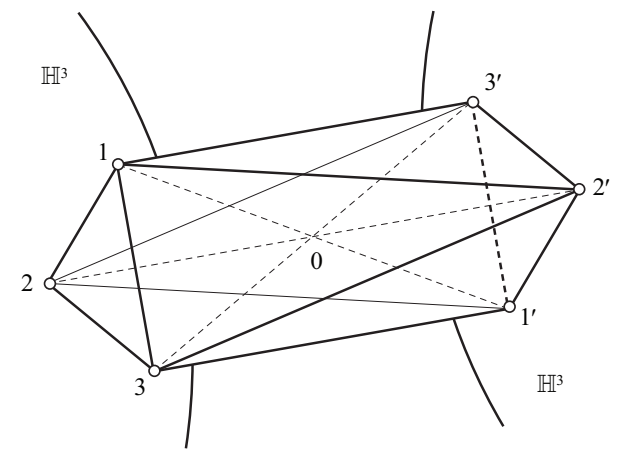

Figure 18

The octahedron obtained above is divided into 8 tetrahedral pieces, which have three ideal vertices and one common vertex 0 . We know that $\operatorname{vol}\left\langle 01^{\prime} 23^{\prime}\right\rangle=$ $\operatorname{vol}\left\langle 012^{\prime} 3\right\rangle$ by symmetry and hence

$$
2 \operatorname{vol} S=2 \operatorname{vol}\langle 0123\rangle=\operatorname{vol}\left\langle 1232^{\prime}\right\rangle+\operatorname{vol}\left\langle 1233^{\prime}\right\rangle-\operatorname{vol}\left\langle 11^{\prime} 23^{\prime}\right\rangle .
$$




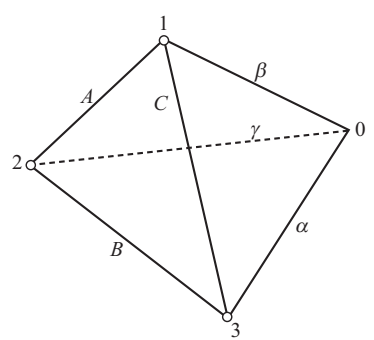

FIGURE 19

Let us denote the dihedral angles of the tetrahedron $S$ as $\alpha, \beta, \gamma, A, B, C$ (see Fig. 19). Note that a $(3,1)$ ideal vertex condition gives another type of ideal vertex condition. For a usual $(4,0)$ ideal tetrahedron, we know the ideal vertex condition $\alpha+\beta+\gamma=\pi$ for the surrounding dihedral angles $\alpha, \beta, \gamma$ at each ideal vertex, and apply this condition to our $(3,1)$ ideal tetrahedron. Then the tetrahedron $S$ has three relations different from the usual one $\alpha+(\pi-B)+$ $(\pi-C)=\pi, \beta+(\pi-A)+(\pi-C)=\pi, \gamma+(\pi-A)+(\pi-B)=\pi$, here we can easily check the relations (see Fig. 20). Therefore, we get

$$
A=\frac{\pi-\alpha+\beta+\gamma}{2}, B=\frac{\pi+\alpha-\beta+\gamma}{2}, C=\frac{\pi+\alpha+\beta-\gamma}{2} .
$$

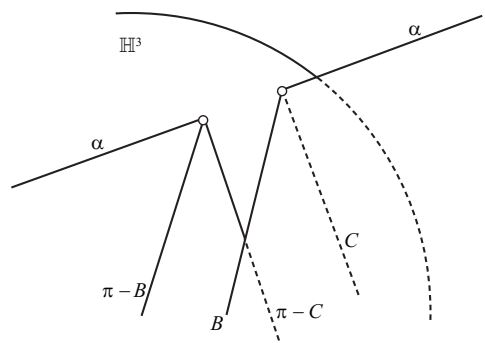

Figure 20

Then the ideal tetrahedron $\left\langle 1232^{\prime}\right\rangle$ has common dihedral angles at vertex 2 with $S$. This $(3,1)$ ideal tetrahedron is obtained as a trihedron minus the $(4,0)$ ideal tetrahedron, where this trihedron is obtained by extending edges $\left\langle 2^{\prime} 1\right\rangle$, $\left\langle 2^{\prime} 2\right\rangle,\left\langle 2^{\prime} 3\right\rangle$, so the $(3,1)$ ideal tetrahedron has the property that the sum of two opposite dihedral angles is $\pi$.

Therefore, the dihedral angles of $\left\langle 1232^{\prime}\right\rangle$ are $\pi-A, \pi-B, \gamma, A, B, \pi-\gamma$ (see Fig. 21), and the volume of the tetrahedron $\left\langle 1232^{\prime}\right\rangle$ is the volume of a trihedron with three dihedral angles $\pi-A, \pi-B, \gamma$ minus an ordinary ideal tetrahedron with three dihedral angles $\pi-A, \pi-B, \gamma$. The volume of the ideal tetrahedron is given by (5) and the volume of the trihedron is given by (7), 

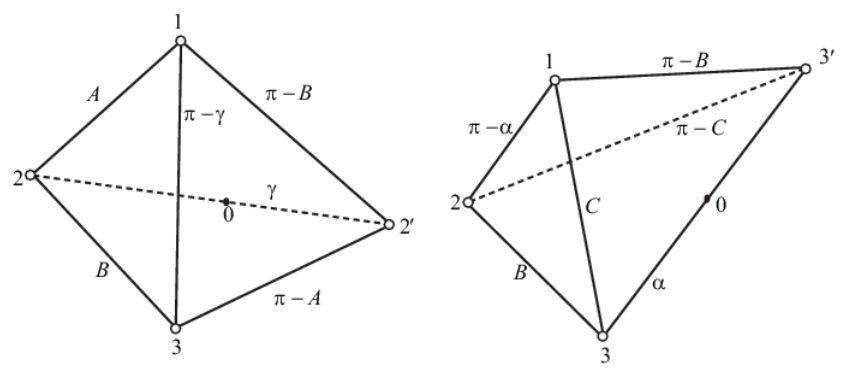

FiguRE 21

so the volume of $\left\langle 1232^{\prime}\right\rangle$ follows as

$$
\begin{aligned}
\operatorname{vol}\left\langle 1232^{\prime}\right\rangle & =\operatorname{vol}(\operatorname{Tri}(\pi-A, \pi-B, \gamma))-\operatorname{vol}(\operatorname{Tetra}(\pi-A, \pi-B, \gamma)) \\
& =\frac{\pi i}{2}(-\pi+A+B-\gamma)-\wedge(\pi-A)-\wedge(\pi-B)-\wedge(\gamma) .
\end{aligned}
$$

Similarly, six dihedral angles of the tetrahedron $\left\langle 1233^{\prime}\right\rangle$ are obtained and represented by $\alpha, \pi-B, \pi-C, \pi-\alpha, B, C$ (see Fig. 21). We can also obtain the volume of $\left\langle 1233^{\prime}\right\rangle$ as

$$
\operatorname{vol}\left\langle 1233^{\prime}\right\rangle=\frac{\pi i}{2}(-\pi+B+C-\alpha)-\wedge(\pi-B)-\wedge(\pi-C)-\wedge(\alpha) .
$$

The $(2,2)$-type ideal tetrahedron $\left\langle 121^{\prime} 3^{\prime}\right\rangle$ requires another method. The angles are easily obtained and represented as $\pi-B, \pi-\beta, \frac{\pi-\alpha-\beta-\gamma}{2}$ using the property that two opposite dihedral angles are the same (see Fig. 22).

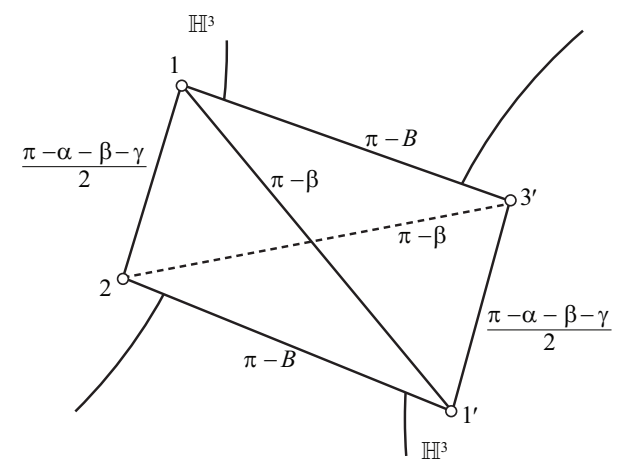

FIGURE 22

Let us consider a general (2,2)-type ideal tetrahedron with dihedral angles $\alpha, \beta, \gamma$, where $\beta, \gamma$ are dihedral angles at the Lorentz part and $\alpha$ is a dihedral 
angles at the hyperbolic part. Then the volume of the $(2,2)$ ideal tetrahedron with dihedral angles $\alpha, \beta, \gamma$ can be deduced from

$$
\operatorname{vol}(\operatorname{Tr} i(\alpha, \beta, \gamma))-\operatorname{vol}(\operatorname{Tr} i(\alpha, \pi-\beta, \pi-\gamma))+\operatorname{vol}(\operatorname{Tetra}(\alpha, \pi-\beta, \pi-\gamma)) .
$$

The exact explanation is shown in Fig. 23.

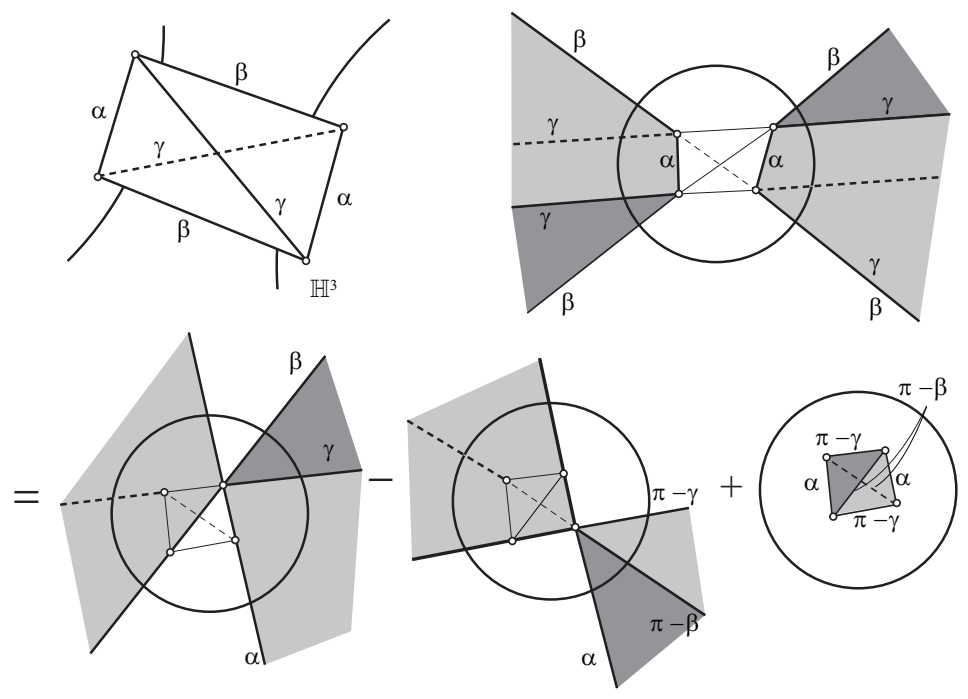

Figure 23

We know that

$$
\begin{aligned}
\operatorname{vol}(\operatorname{Tri}(\alpha, \beta, \gamma)) & =\frac{\pi i}{2}(\pi-\alpha-\beta-\gamma), \\
\operatorname{vol}(\operatorname{Tri}(\alpha, \pi-\beta, \pi-\gamma)) & =\frac{\pi i}{2}(-\pi-\alpha+\beta+\gamma), \\
\operatorname{vol}(\operatorname{Tetr} a(\alpha, \pi-\beta, \pi-\gamma)) & =\wedge(\alpha)+\wedge(\pi-\beta)+\wedge(\pi-\gamma) \\
& =\wedge(\alpha)-\wedge(\beta)-\wedge(\gamma),
\end{aligned}
$$

so we get the volume of the $(2,2)$ ideal tetrahedron as follows:

$$
\begin{aligned}
& \frac{\pi i}{2}(\pi-\alpha-\beta-\gamma)-\frac{\pi i}{2}(-\pi-\alpha+\beta+\gamma)+\wedge(\alpha)-\wedge(\beta)-\wedge(\gamma) \\
= & \pi i(\pi-\beta-\gamma)+\wedge(\alpha)-\wedge(\beta)-\wedge(\gamma) .
\end{aligned}
$$

Since our $(2,2)$ ideal tetrahedron $\left\langle 121^{\prime} 3^{\prime}\right\rangle$ has three dihedral angles $\frac{\pi-\alpha-\beta-\gamma}{2}$, $\pi-B$, and $\pi-\beta$, the volume of $\left\langle 121^{\prime} 3^{\prime}\right\rangle$ is obtained as

$$
\pi i(-\pi+B+\beta)+\wedge\left(\frac{\pi-\alpha-\beta-\gamma}{2}\right)+\wedge(B)+\wedge(\beta) .
$$


Therefore, we conclude that the volume of the tetrahedron $S$ is represented by using the formulas (24), (25), (26), and (23), as follows

$$
\begin{aligned}
2 \operatorname{vol} S= & \operatorname{vol}\left\langle 1232^{\prime}\right\rangle+\operatorname{vol}\left\langle 1233^{\prime}\right\rangle-\operatorname{vol}\left\langle 121^{\prime} 3^{\prime}\right\rangle \\
= & \frac{\pi i}{2}(\pi-\alpha-\beta-\gamma)+\wedge(A)+\wedge(B)+\wedge(C)-\wedge(\alpha)-\wedge(\beta)-\wedge(\gamma) \\
& -\wedge\left(\frac{\pi-\alpha-\beta-\gamma}{2}\right) .
\end{aligned}
$$
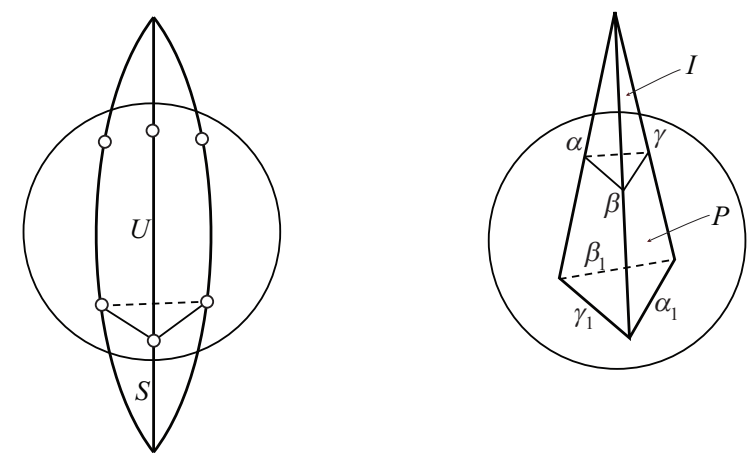

FIGURE 24

If we pull one hyperbolic vertex of a tetrahedron with three ideal vertices out to the Lorentzian part, the tetrahedron has three more ideal points on its edges. So the resulting tetrahedron $U$ is different from the tetrahedron $S$, which has only 3 ideal points on its edges (see Fig. 24). By considering a trihedron with dihedral angles $\alpha, \beta, \gamma$, we can make the tetrahedron $U$ that we really want by eliminating $S$ from $\operatorname{Tr} i(\alpha, \beta, \gamma)$. That tetrahedron $U$ has six dihedral angles $\alpha, \beta, \gamma$, and $\pi-A, \pi-B, \pi-C$, i.e.,

$$
\alpha, \beta, \gamma, \alpha_{1}=\frac{\pi+\alpha-\beta-\gamma}{2}, \beta_{1}=\frac{\pi-\alpha+\beta-\gamma}{2}, \gamma_{1}=\frac{\pi-\alpha-\beta+\gamma}{2} .
$$

Now we can calculate the volume of $U$

$$
\begin{aligned}
2 \operatorname{vol}(U)= & 2 \operatorname{vol}(\operatorname{Tri}(\alpha, \beta, \gamma))-2 \operatorname{vol}(S) \\
= & \frac{\pi i}{2}(\pi-\alpha-\beta-\gamma)+\wedge(\alpha)+\wedge(\beta)+\wedge(\gamma) \\
& +\wedge\left(\frac{\pi+\alpha-\beta-\gamma}{2}\right)+\wedge\left(\frac{\pi-\alpha+\beta-\gamma}{2}\right)+\wedge\left(\frac{\pi-\alpha-\beta+\gamma}{2}\right) \\
& -\wedge\left(\frac{\alpha+\beta+\gamma-\pi}{2}\right) .
\end{aligned}
$$


On the other hand, note that

$$
\begin{aligned}
\operatorname{vol}(U)=\operatorname{vol}(I)+\operatorname{vol}(P) & =\frac{1}{2} \operatorname{vol}(\text { Tri }(\alpha, \beta, \gamma))+\operatorname{vol}(P) \\
& =\frac{\pi i}{4}(\pi-\alpha-\beta-\gamma)+\operatorname{vol}(P)
\end{aligned}
$$

hence we get

$$
2 \operatorname{vol}(P)=\wedge(\alpha)+\wedge(\beta)+\wedge(\gamma)+\wedge\left(\alpha_{1}\right)+\wedge\left(\beta_{1}\right)+\wedge\left(\gamma_{1}\right)-\wedge\left(\frac{\alpha+\beta+\gamma-\pi}{2}\right)
$$

where $\alpha_{1}=\frac{\pi+\alpha-\beta-\gamma}{2}, \beta_{1}=\frac{\pi-\alpha+\beta-\gamma}{2}, \gamma_{1}=\frac{\pi-\alpha-\beta+\gamma}{2}$.

Consequently, formulas (27) and (6) have the same expression, showing the (special case of) a geometric version of Kellerhals's and Usijima's results from the viewpoint of extended hyperbolic space (see [8] and [13]).

Now we will use $\Lambda$ instead of $\wedge$. The two functions $\Lambda$ and $\Lambda$ have the same values for $\alpha, \beta, \gamma, \alpha_{1}, \beta_{1}, \gamma_{1}$, but $\Lambda\left(\frac{\alpha+\beta+\gamma-\pi}{2}\right) \neq \wedge\left(\frac{\alpha+\beta+\gamma-\pi}{2}\right)$. If we pull the non-ideal vertex out to outside $\mathbb{H}^{n}$, then the value $\frac{\alpha+\beta+\gamma-\pi}{2}$ will change from + to - , hence we get the formula (8) for the clockwise contour choice by formula (4).

Consequently, we obtain

$$
2 \operatorname{vol}(U)=\Lambda(\alpha)+\Lambda(\beta)+\Lambda(\gamma)+\Lambda\left(\alpha_{1}\right)+\Lambda\left(\beta_{1}\right)+\Lambda\left(\gamma_{1}\right)-\Lambda\left(\frac{\alpha+\beta+\gamma-\pi}{2}\right)
$$

thereby reproving Theorem 3.2.

Acknowledgments. The authors would like to thank referee's helpful comments.

\section{References}

[1] D. V. Alekseevskij, E. B. Vinberg, and A. S. Solodovnikov, Geometry of Space of Constant Curvature, Geometry, II, 1-138, Encyclopaedia Math. Sci., 29, Springer, Berlin, 1993.

[2] B. Böhm and H. C. Im Hof, Flächeninhalt Verallgemeinerter Hyperbolischer Dreiecke, Geom. Dedicata 42 (1992), no. 2 223-233.

[3] Y. Cho, Trigonometry in extended hyperbolic space and extended de Sitter space, Bull. Korean Math. Soc. 46 (2009), no. 6, 1099-1133.

[4] Y. Cho and H. Kim, On the volume formula for hyperbolic tetrahedra, Discrete Comput. Geom. 22 (1999), no. 3, 347-366.

[5] - Volume of $C^{1, \alpha}$-boundary domain in extended hyperbolic space, J. Korean Math. Soc. 43 (2006), no. 6, 1143-1158.

[6], The analytic continuation of hyperbolic space, Geom. Dedicata 161 (2012), 129-155.

[7] P. Doyle and G. Leibon, 23040 symmetries of hyperbolic tetrahedra, arXiv:math. GT/0309187.

[8] R. Kellerhals, On the volume of hyperbolic polyhedra, Math. Ann. 285 (1989), 541-569.

[9] A. Kolpakov and J. Murakami, Volume of a doubly truncated hyperbolic tetrahedron, arXiv:math.MG/1203.1061v4. 
[10] J. Milnor, Hyperbolic geometry: the first 150 years, Bull. Am. Math. Soc. (N.S.) 6 (1982), no. $1,9-24$.

[11] J. Murakami and M. Yano, On the volume of a hyperbolic and spherical tetrahedron, Comm. Anal. Geom. 13 (2005), no. 2, 379-400

[12] J. G. Ratcliffe, Foundations of Hyperbolic Manifolds, Graduate Texts in Mathematics, 149. Springer-Verlag, New York, 1994

[13] A. Ushijima, A volume formula for generalised hyperbolic tetrahedra, Non-Euclidean geometries, 249-265, Math. Appl. (N. Y.), 581, Springer, New York, 2006.

[14] E. B. Vinberg, Volumes of non-Euclidean Polyhedra, Russian Math. Surveys 48 (1993), no. $2,15-45$.

\section{Yunhi ChO}

Department of Mathematics

University of SEOUL

Seoul 130-743, Korea

E-mail address: yhcho@uos.ac.kr

HYUK KIM

Department of Mathematics

Seoul National University

SEOul 151-742, Korea

E-mail address: hyukkim@snu.ac.kr 